\title{
SIEGEL PARAMODULAR FORMS OF WEIGHT 2 AND SQUAREFREE LEVEL
}

\author{
CRIS POOR, JERRY SHURMAN, AND DAVID S. YUEN
}

\begin{abstract}
We compute the space $\mathcal{S}_{2}(\mathrm{~K}(N))$ of weight 2 Siegel paramodular cusp forms of squarefree level $N<300$. In conformance with the paramodular conjecture of A. Brumer and K. Kramer, the space is only the additive (Gritsenko) lift space of the Jacobi cusp form space $\mathrm{J}_{2, N}^{\text {cusp }}$ except for $N=249,295$, when it further contains one nonlift newform. For these two values of $N$, the Hasse-Weil $p$-Euler factors of a relevant abelian surface match the spin $p$-Euler factors of the nonlift newform for the first two primes $p \nmid N$.
\end{abstract}

\section{INTRODUCTION}

The paramodular conjecture of A. Brumer and K. Kramer BK14 says, in slight paraphrase and restricted to the case of abelian surfaces:

For any positive integer $N$ there is a one-to-one correspondence between isogeny classes of abelian surfaces $A$ over $\mathbb{Q}$ of conductor $N$ with $\operatorname{End}_{\mathbb{Q}}(A)=\mathbb{Z}$, and lines $\mathbb{C} f$ for nonlift degree 2 Siegel paramodular Hecke newforms $f$ of weight 2 and level $N$ having rational eigenvalues. Moreover, the Hasse-Weil L-function of $A$ and the spin L-function of $f$ should match, and the $\ell$-adic representation of $\mathbb{T}_{\ell}(A) \otimes \mathbb{Q}_{\ell}$ should be isomorphic to those associated to $f$ for any $\ell$ prime to $N$.

Here the lift space is $\operatorname{Grit}\left(\mathrm{J}_{2, N}^{\text {cusp }}\right)$, the Gritsenko (additive) lift of the Jacobi cusp form space of weight 2 and index $N$. This lift space lies inside the Siegel paramodular cusp form space $\mathcal{S}_{2}(\mathrm{~K}(N))$ - the subscript 2 indicates the weight, and $\mathrm{K}(N)$ denotes the paramodular group of degree 2 and level $N$; the degree is omitted from the notation because all Siegel modular forms in this article have degree 2. Newforms on $\mathrm{K}(N)$ are by definition Hecke eigenforms orthogonal to the images of level-raising operators from paramodular forms of lower levels [RS07. Notation and terminology will be reviewed in section 2 ,

In [PY15], the first and third authors of this article studied $\mathcal{S}_{2}(\mathrm{~K}(N))$ for prime levels $N<600$. Because $\operatorname{dim} \mathcal{S}_{2}(\mathrm{~K}(N))$ is unknown in general, algorithms were used to bound this dimension by working in $\mathcal{S}_{4}(\mathrm{~K}(N))$, whose dimension is known for prime $N$ by work of T. Ibukiyama [ Ibu85, Ibu07]. The algorithms proved that $\mathcal{S}_{2}(\mathrm{~K}(N))=\operatorname{Grit}\left(\mathrm{J}_{2, N}^{\text {cusp }}\right)$ for all prime $N<600$ other than the exceptional cases $N=277,349,353,389,461,523,587$, precisely the prime $N<600$ for which relevant abelian surfaces exist. Also, $\mathcal{S}_{2}(\mathrm{~K}(277))$ contains one nonlift dimension $\mathbb{C} f_{277}$, and A. Brumer and J. Voight and the first and third authors of this article have shown

Date: July 2, 2018.

2010 Mathematics Subject Classification. Primary: 11F46; secondary: 11F55,11F30,11F50.

Key words and phrases. Siegel paramodular cusp form, Borcherds product. 
that the equality of $L\left(f_{277}, s\right.$, spin $)$ and $L\left(A_{277}, s\right.$, Hasse-Weil $)$ holds conditionally on the existence of certain Galois representations Yue15]. A nonlift eigenform in $\mathcal{S}_{2}(\mathrm{~K}(587))^{-}$has been constructed as well [GPY16]. We are currently working on constructing nonlift forms in the remaining levels.

In BPY16, J. Breeding and the first and third authors of this article showed that $\mathcal{S}_{2}(\mathrm{~K}(N))=\operatorname{Grit}\left(\mathrm{J}_{2, N}^{\text {cusp }}\right)$ for all $N \leq 60$. A key method here was Jacobi restriction, to be described briefly in section 2, and see also [PY13. The article BPY16 established a sufficient number of Fourier-Jacobi coefficients necessary to make Jacobi restriction rigorous at a given level. Running Jacobi restriction to this many coefficients was tractable for levels up to 60 . One idea of the present article is that new algorithms reduce the number of Fourier-Jacobi coefficients known to be sufficient to certify Jacobi restriction, making the number small enough that running Jacobi restriction with that many coefficients is tractable for higher levels.

This article reports our investigation for squarefree composite levels $N<300$, necessarily using different methods from PY15. Among these levels that are also odd, isogeny classes of abelian surfaces exist only for $N=249$ and $N=295$, and at those two levels, the one known isogeny class contains Jacobians of hyperelliptic curves. Specifically, we may take $A_{249}$ to be the Jacobian of $y^{2}=x^{6}+4 x^{5}+$ $4 x^{4}+2 x^{3}+1$ and $A_{295}$ to be the Jacobian of $y^{2}=x^{6}-2 x^{3}-4 x^{2}+1$ [BK14]. Our computations affirm that indeed $\mathcal{S}_{2}(\mathrm{~K}(N))$ contains one nonlift dimension for $N=249,295$, but otherwise is only the lift space $\operatorname{Grit}\left(\mathrm{J}_{2, N}^{\text {cusp }}\right)$. For $N=249,295$, we construct the nonlift eigenform as the sum of a Borcherds product and a Gritsenko lift, and we show that its first two good spin $p$-Euler factors match the Hasse-Weil $p$-Euler factors of the relevant abelian surface. Thus the main result of this article is as follows.

Theorem 1.1. For all composite squarefree $N<300$ except $N=249$ and $N=295$, $\mathcal{S}_{2}(\mathrm{~K}(N))=\operatorname{Grit}\left(\mathrm{J}_{2, N}^{\text {cusp }}\right)$. For $N=249$ and $N=295, \mathcal{S}_{2}(\mathrm{~K}(N))$ contains one nonlift newform dimension $\mathbb{C} f_{N}$ beyond $\operatorname{Grit}\left(\mathrm{J}_{2, N}^{\text {cusp }}\right)$. For the abelian surfaces $A_{249}$ and $A_{295}$ defined in the previous paragraph, the $p$-Euler factor of $L\left(f_{249}, s\right.$, spin $)$ matches the $p$-Euler factor of $L\left(A_{249}, s\right.$, Hasse-Weil $)$ for $p=2$ and $p=5$, and the $p$ Euler factor of $L\left(f_{295}, s\right.$, spin $)$ matches the $p$-Euler factor of $L\left(A_{295}, s\right.$, Hasse-Weil $)$ for $p=2$ and $p=3$.

For the level 249 nonlift newform, let $\vartheta(\tau, z)$ be Jacobi's odd theta function and let $\vartheta_{r}(\tau, z)=\vartheta(\tau, r z)$ for $r \in \mathbb{Z}_{\geq 1}$, and introduce a product of quotients of theta functions,

$$
\psi_{249}=\frac{\vartheta_{8}}{\vartheta_{1}} \frac{\vartheta_{18}}{\vartheta_{6}} \frac{\vartheta_{14}}{\vartheta_{7}} .
$$

The nonlift newform of $\mathcal{S}_{2}(\mathrm{~K}(249))$ is then

$$
\begin{aligned}
f_{249}= & 14 \operatorname{Borch}\left(\psi_{249}\right) \\
& -6 \operatorname{Grit}(\operatorname{TB}(2 ; 2,3,3,4,5,6,7,9,10,13)) \\
& -3 \operatorname{Grit}(\operatorname{TB}(2 ; 2,2,3,5,5,6,7,9,11,12)) \\
& +3 \operatorname{Grit}(\operatorname{TB}(2 ; 1,3,3,5,6,6,6,9,11,12)) \\
& +2 \operatorname{Grit}(\operatorname{TB}(2 ; 1,1,2,3,4,5,6,9,10,15)) \\
& +7 \operatorname{Grit}(\operatorname{TB}(2 ; 1,2,3,3,4,5,6,9,11,14)),
\end{aligned}
$$


where "Borch" and "Grit" and "TB" respectively denote the Borcherds product, the Gritsenko lift, and the theta block construction. This will be explained further in section 8. Thus $f_{249}$ is congruent to a Gritsenko lift modulo 14, and we note that the two isogenous Jacobians of genus 2 curves of conductor 249 defined over $\mathbb{Q}$ have torsion groups $\mathbb{Z} / 14 \mathbb{Z}$ and $\mathbb{Z} / 28 \mathbb{Z}$, as shown at $\operatorname{lmfdb.org.~}$

Again with $\operatorname{dim} \mathcal{S}_{2}(\mathrm{~K}(N))$ unavailable, we used algorithms to bound this dimension by working in $\mathcal{S}_{4}(\mathrm{~K}(N))$, whose dimension is known by work of T. Ibukiyama and H. Kitayama [K]. These algorithms require spanning most of the weight 4 space but not necessarily all of it: the requisite spanned subspace can fall short essentially by the dimension of the Jacobi cusp form space $\mathrm{J}_{2, N}^{\text {cusp }}$, which is known by work of N. Skoruppa and D. Zagier [EZ85, SZ89. Thus our major computational challenge was to span enough of $\mathcal{S}_{4}(\mathrm{~K}(N))$. This space presents various impediments to diverse spanning methods, so we had to employ a range of approaches. The methods that worked for prime $N$ generally did not help for composite squarefree $N$ : Hecke spreading, which spanned the Fricke plus space for large prime levels, is obstructed for composite levels by the various Atkin-Lehner signatures that are possible; and theta tracing, which spanned the Fricke plus space for small prime levels and the Fricke minus space for large prime levels, appears to be more expensive computationally for composite levels. For composite squarefree $N$, our methods are to trace $\left(\operatorname{Grit}\left(\mathrm{J}_{2, N q}^{\text {cusp }}\right)\right)^{2}$ and $\operatorname{Grit}\left(\mathrm{J}_{4, N q}^{\text {cusp }}\right)$ down to level $N$ from level $N q$ for a small prime $q$ that does not divide $N$, to Hecke spread $\left(\operatorname{Grit}\left(J_{2, N}^{\text {cusp }}\right)\right)^{2}$ at level $N$, and to compute Borcherds products in the Fricke plus and minus spaces at level $N$. When enough of the weight 4 space is spanned, the algorithms for weight 2 show that Jacobi restriction computations with only a small number of Fourier-Jacobi coefficients are rigorous, and these computations give the results.

See the website Yue16] for reports on the computations that this article discusses. For example, we sketch the online report for level $N=286$, which the reader could examine alongside this paragraph. The space $\mathcal{S}_{4}(\mathrm{~K}(286))$ has 189 dimensions, of which the lift space $\operatorname{Grit}\left(\mathrm{J}_{4,286}^{\mathrm{cusp}}\right)$ comprises 48 . Jacobi restriction heuristically finds 113 more Fricke plus space dimensions, giving 161 Fricke plus space dimensions altogether, and 28 Fricke minus space dimensions. This heuristic information is essential for targeting our constructions, e.g., deciding when to switch from one method to another. Tracing the weight 4 Gritsenko lifts and the twofold products of weight 2 Gritsenko lifts from level $1430=286 \cdot 5$ down to level 286 and then adding the twofold products of weight 2 Gritsenko lifts at level 286 gives 157 plus space dimensions and no minus space dimensions. Hecke spreading gives 8 minus dimensions. Adding in Borcherds products raises the spanned plus and minus space dimensions to 161 and 27 , so one dimension is missing and we think that it lies in the minus space. This gives enough of the weight 4 space to run our weight 2 diagnostic tests, to be described in section 4. The $H_{4}(286,3,1)^{+}$test says that weight 2 Jacobi restriction to two or more terms gives a dimension upper bound of the Fricke plus space $\mathcal{S}_{2}(\mathrm{~K}(286))^{+}$. Thus, weight 2 Jacobi restriction to five terms, which we have carried out, correctly bounds the dimension of the Fricke plus space by 3 , the dimension of the lift space Grit $\left(\mathrm{J}_{2,286}^{\text {cusp }}\right)$; this shows that these two spaces are equal. The $H_{4}(286,1,1)^{-}$test says that the Fricke minus space is 0 . So altogether $\mathcal{S}_{2}(\mathrm{~K}(286))$ is the lift space. Whereas the $H_{4}(286,3,1)^{+}$test says that Jacobi restriction to two or more terms gives a Fricke plus space dimension upper bound, the theoretical bound used in [BPY16] says this only for 24 or 
more terms. This improvement is crucial: running Jacobi restriction systematically across many levels to the number of terms required by the bound used in BPY16] is computationally unviable for now.

The Borcherds products used in the computation at level $N=286$ are given at the website [Yue16. For the Fricke plus space, the relevant file at the website explains that 60 Borcherds products $\operatorname{Borch}(\psi)$ were used to find the additional four dimensions reported; each $\psi$ lies in the space $\mathrm{J}_{0,286}^{\mathrm{w} . \mathrm{h}}$ of weight 0 , index 286 weakly holomorphic Jacobi forms, and is a linear combination of a basis of $\mathrm{J}_{12,286}^{\text {cusp }} / \Delta_{12}$ where $\Delta_{12} \in \mathrm{J}_{12,0}^{\text {cusp }}$ is the classical discriminant function. The basis of $\mathrm{J}_{12,286}^{\text {cusp }} / \Delta_{12}$, built from theta blocks, is given at the end of the file. The website's file for the Fricke minus space is similar, explaining that 22 Borcherds products $\operatorname{Borch}(\psi)$ were used to find the additional 19 dimensions reported, with each $\psi$ now the sum of a quotient $\phi \mid V_{2} / \phi$ and a linear combination of the basis of $\mathrm{J}_{12,286}^{\text {cusp }} / \Delta_{12}$; here $\phi$ is a theta block and $V_{2}$ is an index-raising Hecke operator. Our Borcherds product files will be described further in section 7

Section 2 gives background for this article. Section 3 shows that for low or odd weight and squarefree level, either all Siegel paramodular forms are cusp forms or the vanishing of a Siegel paramodular form's constant term suffices to make it a cusp form. Section 4 establishes the algorithms that study weight 2 Siegel paramodular cusp forms by working in weight 4 . Section 5 describes our tracing down method, and section 6 describes our use of Hecke spreading. Section 7 gives a result that certain conditions suffice for a Borcherds product of low weight and squarefree level to be a Siegel paramodular cusp form. Finally, section 8 describes how we used this result to construct the weight 2 nonlift newforms at levels $N=249$ and $N=295$.

We thank Fordham University for letting us carry out computations on its servers. We thank Reed College for making its computer lab machines available to us, and especially the second author thanks B. Salzberg for helping him use them in parallel.

\section{BACKGROUND}

We introduce notation and terminology for Siegel paramodular forms. The degree 2 symplectic group $\mathrm{Sp}(2)$ of $4 \times 4$ matrices is defined by the condition $g^{\prime} J g=J$, where the prime denotes matrix transpose and $J$ is the skew form $\left[\begin{array}{rr}0 & -1 \\ 1 & 0\end{array}\right]$ with each block $2 \times 2$. The Klingen and Siegel parabolic subgroups of $\operatorname{Sp}(2)$ are respectively

$$
\mathrm{P}_{2,1}=\left\{\left[\begin{array}{cc|cc}
* & 0 & * & * \\
* & * & * & * \\
\hline * & 0 & * & * \\
0 & 0 & 0 & *
\end{array}\right]\right\}, \quad \mathrm{P}_{2,0}=\left\{\left[\begin{array}{cc|cc}
* & * & * & * \\
* & * & * & * \\
\hline 0 & 0 & * & * \\
0 & 0 & * & *
\end{array}\right]\right\}
$$

For $\mathrm{P}_{2,1}$, the three zeros on the bottom row force the other two zeros in consequence of the matrices being symplectic. For any positive integer $N$, the paramodular group $\mathrm{K}(N)$ of degree 2 and level $N$ is the group of rational symplectic matrices that stabilize the column vector lattice $\mathbb{Z} \oplus \mathbb{Z} \oplus \mathbb{Z} \oplus N \mathbb{Z}$. In coordinates,

$$
\mathrm{K}(N)=\left\{\left[\begin{array}{cc|cc}
* & * N & * & * \\
* & * & * & * / N \\
\hline * & * N & * & * \\
* N & * N & * N & *
\end{array}\right] \in \mathrm{Sp}_{2}(\mathbb{Q}): \text { all } * \text { entries integral }\right\} .
$$


Here the upper right entries of the four subblocks are "more integral by a factor of $N$ " than implied immediately by the definition of the paramodular group as a lattice stabilizer, but as with $\mathrm{P}_{2,1}$ the extra conditions hold because the matrices are symplectic.

Let $\mathcal{H}_{2}$ denote the Siegel upper half space of $2 \times 2$ symmetric complex matrices that have positive definite imaginary part. Elements of this space are notated

$$
\Omega=\left[\begin{array}{cc}
\tau & z \\
z & \omega
\end{array}\right] \in \mathcal{H}_{2}
$$

and also, letting $\mathrm{e}(z)=e^{2 \pi i z}$ for $z \in \mathbb{C}$, the notation

$$
q=\mathrm{e}(\tau), \quad \zeta=\mathrm{e}(z), \quad \xi=\mathrm{e}(\omega)
$$

is standard. The real symplectic group $\operatorname{Sp}_{2}(\mathbb{R})$ acts on $\mathcal{H}_{2}$ via fractional linear transformations, $g(\Omega)=(a \Omega+b)(c \Omega+d)^{-1}$ for $g=\left[\begin{array}{ll}a & b \\ c & d\end{array}\right]$, and the factor of automorphy is $j(g, \Omega)=\operatorname{det}(c \Omega+d)$. Fix an integer $k$. Any function $f: \mathcal{H}_{2} \longrightarrow \mathbb{C}$ and any real symplectic matrix $g \in \mathrm{Sp}_{2}(\mathbb{R})$ combine to form another such function through the weight $k$ operator, $f[g]_{k}(\Omega)=j(g, \Omega)^{-k} f(g(\Omega))$. A Siegel paramodular form of weight $k$ and level $N$ is a holomorphic function $f: \mathcal{H}_{2} \longrightarrow \mathbb{C}$ that is $[\mathrm{K}(N)]_{k^{-}}$ invariant; the Köcher Principle says that for any positive $2 \times 2$ real matrix $Y_{o}$, the function $f[g]_{k}$ is bounded on $\left\{\operatorname{Im}(\Omega)>Y_{o}\right\}$ for all $g \in \operatorname{Sp}_{2}(\mathbb{Q})$. The space of weight $k$, level $N$ Siegel paramodular forms is denoted $\mathcal{M}_{k}(\mathrm{~K}(N))$. Dimension formula methods for $\mathcal{M}_{k}(\mathrm{~K}(N))$ based on the Riemann-Roch Theorem or trace formulas are not available for $k=2$.

The Witt map $\iota^{*}$ takes functions $f: \mathcal{H}_{2} \longrightarrow \mathbb{C}$ to functions $\iota^{*} f: \mathcal{H} \times \mathcal{H} \longrightarrow \mathbb{C}$, with $\left(\iota^{*} f\right)(\tau \times \omega)=f\left(\left[\begin{array}{cc}\tau & 0 \\ 0 & \omega\end{array}\right]\right)$. Especially, the Witt map takes $\mathcal{M}_{k}(\mathrm{~K}(N))$ to $\mathcal{M}_{k}\left(\mathrm{SL}_{2}(\mathbb{Z})\right) \otimes \mathcal{M}_{k}\left(\mathrm{SL}_{2}(\mathbb{Z})\right)\left[\left[\begin{array}{cc}N & 0 \\ 0 & 1\end{array}\right]\right]_{k}$. Siegel's $\Phi$ map takes any holomorphic function that has a Fourier series of the form $f(\Omega)=\sum_{t} a(t ; f) \mathrm{e}(\langle t, \Omega\rangle)$, summing over matrices $t=\left[\begin{array}{ll}n & r \\ r & m\end{array}\right]$ with $n, m \in \mathbb{Q}_{\geq 0}, r \in \mathbb{Q}$, and $n m-r^{2} \geq 0$, to the function $(\Phi f)(\tau)=\lim _{\omega \rightarrow i \infty}\left(\iota^{*} f\right)(\tau, \omega)$. A Siegel paramodular form $f$ in $\mathcal{M}_{k}(\mathrm{~K}(N))$ is a cusp form if $\Phi\left(f[g]_{k}\right)=0$ for all $g \in \mathrm{Sp}_{2}(\mathbb{Q})$; the space of such forms is denoted $\mathcal{S}_{k}(\mathrm{~K}(N))$. The dimension of $\mathcal{S}_{k}(\mathrm{~K}(N))$ is known for squarefree $N$ and $k \geq 3$ Ibu85, Ibu07, IK].

Every Siegel paramodular form of weight $k$ and level $N$ has a Fourier expansion

$$
f(\Omega)=\sum_{t \in \mathcal{X}_{2}(N)^{\mathrm{semi}}} a(t ; f) \mathrm{e}(\langle t, \Omega\rangle)
$$

where $\mathcal{X}_{2}(N)^{\text {semi }}=\left\{\left[\begin{array}{cc}n & r / 2 \\ r / 2 & m N\end{array}\right]: n, m \in \mathbb{Z}_{\geq 0}, r \in \mathbb{Z}, 4 n m N-r^{2} \geq 0\right\}$ and $\langle t, \Omega\rangle=\operatorname{tr}(t \Omega)$. A Siegel paramodular form is a cusp form if and only if its Fourier expansion is supported on $\mathcal{X}_{2}(N)$, defined by the strict inequality $4 n m N-r^{2}>0$; this description of cusp forms does not hold in general for groups commensurable with $\mathrm{Sp}_{2}(\mathbb{Z})$, but it does hold for $\mathrm{K}(N)$. Consider any $\mathrm{Sp}_{2}(\mathbb{R})$ matrix of the form $g=d^{*} \boxplus d=\left[\begin{array}{cc}d^{*} & 0 \\ 0 & d\end{array}\right]$ with $d \in \mathrm{GL}_{2}(\mathbb{R})$, where the superscript asterisk denotes matrix inverse-transpose. Introduce the notation $t[u]=u^{\prime} t u$ for compatibly sized matrices $t$ and $u$. Then we have $f[g]_{k}(\Omega)=(\operatorname{det} d)^{-k} \sum_{t \in \mathcal{X}_{2}(N)^{\operatorname{semi}}\left[d^{*}\right]} a\left(t\left[d^{\prime}\right] ; f\right) \mathrm{e}(\langle t, \Omega\rangle)$ for any Siegel paramodular form $f$, and especially if $g$ normalizes $\mathrm{K}(N)$ so that $f[g]_{k}$ is again a Siegel paramodular form then $a\left(t ; f[g]_{k}\right)=(\operatorname{det} d)^{-k} a\left(t\left[d^{\prime}\right] ; f\right)$ for $t \in \mathcal{X}_{2}(N)^{\text {semi }}$. Let $\Gamma_{ \pm}^{0}(N)$ denote the subgroup of $\mathrm{GL}_{2}(\mathbb{Z})$ defined by the condition $b=0 \bmod N$. For $d \in \Gamma_{ \pm}^{0}(N)$, the matrix $g=d^{-1} \boxplus d^{\prime}$ lies in $\mathrm{K}(N)$ and 
we get $a(t[d] ; f)=(\operatorname{det} d)^{k} a(t ; f)$ for $t \in \mathcal{X}_{2}(N)^{\text {semi }}$. Our programs handle Fourier coefficient indices at the level of $\Gamma_{ \pm}^{0}(N)$-equivalence classes, each class having a canonical representative of the form $t_{o} \times v_{o}$ with $t_{o} \in \mathcal{X}_{2}(1)^{\text {semi }}$ Legendre reduced and with $v_{o} \in \mathbb{Z} / N \mathbb{Z} \oplus \mathbb{Z} / N \mathbb{Z}$.

For each positive divisor $c$ of $N$ such that $\operatorname{gcd}(c, N / c)=1$, let $\hat{c}$ be a multiplicative inverse of $N / c$ modulo $c$. Introduce the $c$-th cusp representative matrix $r_{c}$ and a translation matrix $\beta_{c}$ and their product, the $c$-th Atkin-Lehner matrix $\alpha_{c}=r_{c} \beta_{c}$,

$$
r_{c}=\left[\begin{array}{cc}
1 & 0 \\
N / c & 1
\end{array}\right], \quad \beta_{c}=\frac{1}{\sqrt{c}}\left[\begin{array}{rr}
c & -\hat{c} \\
0 & 1
\end{array}\right], \quad \alpha_{c}=\frac{1}{\sqrt{c}}\left[\begin{array}{cc}
c & -\hat{c} \\
N & 1-(N / c) \hat{c}
\end{array}\right] .
$$

The Atkin-Lehner matrix normalizes the level $N$ Hecke subgroup $\Gamma_{0}(N)$ of $\mathrm{SL}_{2}(\mathbb{Z})$, and it squares into $\Gamma_{0}(N)$; these properties hold for all of $\Gamma_{0}(N) \alpha_{c} \Gamma_{0}(N)$, any of which may be taken as $\alpha_{c}$ instead. For $c=1$ we take $r_{1}=\beta_{1}=\alpha_{1}=$ $1_{2}$. For $c=N$ we modify the Atkin-Lehner matrix, multiplying it from the right by $\left[\begin{array}{ll}1 & 0 \\ N & 1\end{array}\right]$ to get the traditional Fricke involution, $\alpha_{N}=\frac{1}{\sqrt{N}}\left[\begin{array}{cc}0 & -1 \\ N & 0\end{array}\right]: \tau \mapsto$ $-\frac{1}{N \tau}$. Each Atkin-Lehner matrix $\alpha_{c}$ has a corresponding paramodular AtkinLehner matrix $\mu_{c}=\alpha_{c}^{*} \boxplus \alpha_{c}$ that normalizes $\mathrm{K}(N)$ and squares into $\mathrm{K}(N)$. For $c=1$ we take $\mu_{1}=1_{4}$. For $c=N$, the paramodular Fricke involution is $\mu_{N}=\frac{1}{\sqrt{N}}\left(\left[\begin{array}{cc}0 & -N \\ 1 & 0\end{array}\right] \boxplus\left[\begin{array}{cc}0 & -1 \\ N & 0\end{array}\right]\right):\left[\begin{array}{ll}\tau & z \\ z & \omega\end{array}\right] \longmapsto\left[\begin{array}{cc}\omega N & -z \\ -z & \tau / N\end{array}\right]$. The space $\mathcal{S}_{k}(\mathrm{~K}(N))$ decomposes as the direct sum of the Fricke eigenspaces for the two eigenvalues \pm 1 , $\mathcal{S}_{k}(\mathrm{~K}(N))=\mathcal{S}_{k}(\mathrm{~K}(N))^{+} \oplus \mathcal{S}_{k}(\mathrm{~K}(N))^{-}$. More generally the Atkin-Lehner involutions satisfy $\left[\alpha_{c}\right]_{k}\left[\alpha_{\tilde{c}}\right]_{k}=\left[\alpha_{c \tilde{c}}\right]_{k}$ for coprime divisors $c$ and $\tilde{c}$ of $N$, and so they commute. Thus $\mathcal{S}_{k}(\mathrm{~K}(N))$ decomposes as a direct sum of spaces $\mathcal{S}_{k}(\mathrm{~K}(N))^{v}$ where $v$ is a vector of \pm entries indexed by the prime divisors of the level $N$. Such a vector is called an Atkin-Lehner signature.

The Fourier-Jacobi expansion of a Siegel paramodular cusp form $f \in \mathcal{S}_{k}(\mathrm{~K}(N))$ is

$$
f(\Omega)=\sum_{m \geq 1} \phi_{m}(f)(\tau, z) \xi^{m N}, \quad \Omega=\left[\begin{array}{cc}
\tau & z \\
z & \omega
\end{array}\right], \xi=\mathrm{e}(\omega)
$$

with Fourier-Jacobi coefficients

$$
\phi_{m}(f)(\tau, z)=\sum_{t=\left[\begin{array}{rr}
n & r / 2 \\
r / 2 & m N
\end{array}\right] \in \mathcal{X}_{2}(N)} a(t ; f) q^{n} \zeta^{r}, \quad q=\mathrm{e}(\tau), \zeta=\mathrm{e}(z) .
$$

Here the coefficient $a(t ; f)$ is also written $c\left(n, r ; \phi_{m}\right)$. Each Fourier-Jacobi coefficient $\phi_{m}(f)$ lies in the space $\mathrm{J}_{k, m N}^{\text {cusp }}$ of weight $k$, index $m N$ Jacobi cusp forms, whose dimension is known (for the theory of Jacobi forms, see [EZ85, GN98, SZ89]). These are Jacobi forms of level one - this is an advantage of the paramodular group over the Hecke subgroup $\Gamma_{0}^{(2)}(N)$ of $\operatorname{Sp}_{2}(\mathbb{Z})$ - and trivial character, both omitted from the notation. The additive (Gritsenko) lift Grit : $\mathrm{J}_{k, N}^{\text {cusp }} \longrightarrow \mathcal{S}_{k}(\mathrm{~K}(N))^{\epsilon} \subset \mathcal{S}_{k}(\mathrm{~K}(N))$ for $\epsilon=(-1)^{k}$ is a section of the map $\mathcal{S}_{k}(\mathrm{~K}(N)) \longrightarrow \mathrm{J}_{k, N}^{\text {cusp }}$ that takes each $f$ to $\phi_{1}(f)$, i.e., $\phi_{1}(\operatorname{Grit}(\phi))=\phi$ for all $\phi \in \mathrm{J}_{k, N}^{\text {cusp }}$.

Jacobi restriction is described briefly in section 5 of [BPY16, and we sketch it here as well. Taking an even weight $k$ for simplicity, the coefficients of a Siegel paramodular Fricke eigenform $f \in \mathcal{S}_{k}(\mathrm{~K}(N))^{\epsilon}$ satisfy the Siegel consistency relations $c\left(n, r ; \phi_{m}\right)=c\left(n^{\prime}, r^{\prime} ; \phi_{m^{\prime}}\right)$ for $\left[\begin{array}{cc}n^{\prime} & r^{\prime} / 2 \\ r^{\prime} / 2 & m^{\prime} N\end{array}\right] \in\left[\begin{array}{cc}n & r / 2 \\ r / 2 & m N\end{array}\right]\left[\Gamma_{ \pm}^{0}(N)\right]$, and they satisfy the Fricke eigenform relations $c\left(n, r ; \phi_{m}\right)=\epsilon c\left(m,-r ; \phi_{n}\right)$. For a chosen value 
$m_{\max }$, define a subspace $V\left(m_{\max }\right)$ of $\bigoplus_{m=1}^{m_{\max }} \mathrm{J}_{k, m N}^{\text {cusp }}$ by imposing any subset of the just-mentioned linear relations on the coefficients $c\left(n, r ; \phi_{m}\right)$ with $m \leq m_{\max }$. Thus $V\left(m_{\max }\right)$ contains the image of the map $\mathcal{S}_{k}(\mathrm{~K}(N))^{\epsilon} \longrightarrow \bigoplus_{m=1}^{m_{\max }} \mathrm{J}_{k, m N}^{\mathrm{cusp}}$ that takes each $f$ to $\left(\phi_{1}(f), \ldots, \phi_{m_{\max }}(f)\right)$. In particular, $\operatorname{dim} \mathcal{S}_{k}(\mathrm{~K}(N))^{\epsilon} \leq \operatorname{dim} V\left(m_{\max }\right)$ for $m_{\max }$ large enough to make the map inject; theoretical estimates for $m_{\max }$ in BPY16] guarantee injectivity, but they can be too big for practical use. For values of $k$ and improved values of $m_{\max }$ relevant to this article, we can span the spaces $\mathrm{J}_{k, m N}^{\text {cusp }}$ for $m \leq m_{\max }$ with theta blocks, and so we can compute $\operatorname{dim} V\left(m_{\max }\right)$. For any prime $q$, if we have bases of the Jacobi form spaces over the field $\mathbb{F}_{q}$ of $q$ elements then the same computation modulo $q$ gives the bound $\operatorname{dim} \mathcal{S}_{k}(\mathrm{~K}(N))^{\epsilon} \leq$ $\operatorname{dim}_{\mathbb{F}_{q}} V\left(m_{\text {max }}\right)$. Jacobi restriction is remarkably tractable, and it often gives optimal dimension upper bounds for values of $m_{\max }$ much smaller than the theoretical estimates. Even when we don't know that $m_{\max }$ is large enough to guarantee the bounds given by Jacobi restriction, those bounds are still very useful heuristic estimates. For example, our weight 4 computations that made up the bulk of the project being described in this article were not viable until we used such estimates from Jacobi restriction to decide how many Fourier coefficients the computations should track, and also our work in weight 4 at a given level often involved a confident decision between searching for more Fricke plus space dimensions or more Fricke minus space dimensions based on the heuristic dimensions of the two eigenspaces.

The Dedekind eta function and the odd Jacobi theta function are

$$
\begin{aligned}
\eta(\tau) & =q^{1 / 24} \prod_{n \in \mathbb{Z}_{\geq 1}}\left(1-q^{n}\right), \\
\vartheta(\tau, z) & =\sum_{n \in \mathbb{Z}}(-1)^{n} q^{(n+1 / 2)^{2} / 2} \zeta^{n+1 / 2} .
\end{aligned}
$$

Let $\vartheta_{r}(\tau, z)=\vartheta(\tau, r z)$ for any $r \in \mathbb{Z}_{\geq 1}$. Quotients $\vartheta_{r} / \eta$ are the basic ingredients of the theta block "without denominator" (see GSZ]) associated to any finitely supported function $\varphi: \mathbb{Z}_{\geq 0} \longrightarrow \mathbb{Z}$ with $\varphi(r) \geq 0$ for $r \geq 1$,

$$
\operatorname{TB}(\varphi)(\tau, z)=\eta(\tau)^{\varphi(0)} \prod_{r \in \mathbb{Z}_{\geq 1}}\left(\vartheta_{r}(\tau, z) / \eta(\tau)\right)^{\varphi(r)} .
$$

Any such theta block transforms as a Jacobi form of weight $k=\frac{1}{2} \varphi(0)$ and of index $m=\frac{1}{2} \sum_{r \in \mathbb{Z}_{\geq 1}} r^{2} \varphi(r)$, and when $\frac{1}{24} \varphi(0)+\frac{1}{12} \sum_{r \in \mathbb{Z}_{\geq 1}} \varphi(r) \in \mathbb{Z}$ it has trivial character. The theta block $\operatorname{TB}(\varphi)$ needn't be a Jacobi cusp form, but the "without denominator" stipulation that $\varphi(r) \geq 0$ for $r \geq 1$ makes it lie in the space J.h. of weakly holomorphic weight $k$, index $m$ Jacobi forms, whose Fourier expansions $\psi(\tau, z)=\sum_{n, r} c(n, r) q^{n} \zeta^{r}$ are supported on $n \gg-\infty$. We show that equivalently, the support can be taken to be $4 n m-r^{2} \gg-\infty$. The index $m$ Jacobi form transformation law $\psi(\tau, \lambda \tau+z) q^{\lambda^{2} m} \zeta^{2 \lambda m}=\psi(\tau, z)$ for any $\lambda \in \mathbb{Z}$ shows that $c\left(n-\lambda r+\lambda^{2} m, r-2 \lambda m\right)=c(n, r)$ for all $(n, r)$ and $\lambda$, and also $4\left(n-\lambda r+\lambda^{2} m\right) m-$ $(r-2 \lambda m)^{2}=4 n m-r^{2}$. Thus, for a given value of $4 n m-r^{2}$ we may consider only coefficients $c(n, r)$ with $|r| \leq m$. If for some $n_{o}$, all coefficients $c(n, r)$ where $n<n_{0}$ are 0, then all coefficients $c(n, r)$ where $4 n m-r^{2}<4 n_{o} m-m^{2}$ are 0 ; indeed, we may take $|r| \leq m$, giving $4 n m-m^{2} \leq 4 n m-r^{2}<4 n_{o} m-m^{2}$ and thus $n<n_{o}$, so $c(n, r)=0$ as claimed. Conversely, if for some $d_{0}$, all coefficients $c(n, r)$ where $4 n m-r^{2}<d_{o}$ are 0 , then also $c(n, r)=0$ for all $n<d_{o} /(4 m)$. Thus the weight $k$, index $m$ weakly holomorphic Jacobi forms can be defined by 
the condition $c(n, r)=0$ either for $n \gg-\infty$ or for $4 n m-r^{2} \gg-\infty$, as claimed. Furthermore, given a weakly holomorphic Jacobi form of index $m$, its coefficients $c(n, r)$ where $4 n m-r^{2} \leq 0$ are entirely determined by the finitely many such coefficients indexed by $(n, r)$ such that $n \leq m / 4$ and $|r| \leq m$. This holds because $c(n, r)=c(\tilde{n}, \tilde{r})$ for some $(\tilde{n}, \tilde{r})$ with $|\tilde{r}| \leq m$ and $4 \tilde{n} m-\tilde{r}^{2}=4 n m-r^{2}$; thus $4 \tilde{n} m-m^{2} \leq 4 \tilde{n} m-\tilde{r}^{2}=4 n m-r^{2} \leq 0$, and the claimed inequality $\tilde{n} \leq m / 4$ follows.

Some functions $\varphi: \mathbb{Z}_{\geq 0} \longrightarrow \mathbb{Z}$ that don't take $\mathbb{Z}_{\geq 1}$ to $\mathbb{Z}_{\geq 0}$ still produce weakly holomorphic Jacobi forms under the formula in the previous display. These are theta blocks "with denominator." Our algorithm and program to find Borcherds products to help span spaces $\mathcal{S}_{4}(\mathrm{~K}(N))$ involved theta blocks without denominator, while our construction of the nonlifts in $\mathcal{S}_{2}(\mathrm{~K}(N))$ for $N=249,295$ used theta blocks with denominator.

\section{Cuspidality for Low Or Odd Weight and Squarefree Level}

Our computation used the following cuspidality test. Specifically, the test will be used in the proof of Corollary 7.2, which identifies some Borcherds products as paramodular cusp forms.

Proposition 3.1. Let $N$ be a squarefree positive integer, and let $k$ be a positive integer. If $k=2$ or $k$ is odd then $\mathcal{M}_{2}(\mathrm{~K}(N))=\mathcal{S}_{2}(\mathrm{~K}(N))$. If $k=4,6,8,10,14$ then for all $f \in \mathcal{M}_{k}(\mathrm{~K}(N)), f \in \mathcal{S}_{k}(\mathrm{~K}(N))$ if and only if $a(0 ; f)=0$.

Proof. Recall the matrices $r_{c}, \alpha_{c}, \beta_{c}$, and $\mu_{c}=\alpha_{c}^{*} \boxplus \alpha_{c}$ from section 2 For any squarefree positive integer $N$, every divisor $c$ of $N$ satisfies the Atkin-Lehner condition $\operatorname{gcd}(c, N / c)=1$, and so H. Reefschläger's decomposition (Ree73], and see Theorem 1.2 of [PY13] $\mathrm{Sp}_{2}(\mathbb{Q})=\bigsqcup_{0<c \mid N} \mathrm{~K}(N)\left(r_{c}^{*} \boxplus r_{c}\right) \mathrm{P}_{2,1}(\mathbb{Q})$ combines with the relations $\alpha_{c}=r_{c} \beta_{c}$ to give $\operatorname{Sp}_{2}(\mathbb{Q})=\bigsqcup_{0<c \mid N} \mathrm{~K}(N) \mu_{c}\left(\beta_{c}^{\prime} \boxplus \beta_{c}^{-1}\right) \mathrm{P}_{2,1}(\mathbb{Q})$. Thus any $g \in \mathrm{Sp}_{2}(\mathbb{Q})$ is $g=\kappa \mu_{c} u_{c}$ where $\kappa \in \mathrm{K}(N)$ and $0<c \mid N$ and $u_{c} \in \mathrm{P}_{2,1}(\mathbb{Q}(\sqrt{N / c}))$, and consequently $\Phi\left(f[g]_{k}\right)=\Phi\left(f\left[\mu_{c}\right]_{k}\left[u_{c}\right]_{k}\right)$. Let $u_{1, c} \in \mathrm{GL}_{2}(\mathbb{R})$ denote the $2 \times 2$ matrix of upper left entries of the four blocks of $u_{c}$. For any $f \in \mathcal{M}_{k}(\mathrm{~K}(N))$, a computation shows that $\Phi\left(f\left[\mu_{c}\right]_{k}\left[u_{c}\right]_{k}\right)=\left(m \Phi\left(f\left[\mu_{c}\right]_{k}\right)\right)\left[u_{1, c}\right]_{k}$, where $m$ is a nonzero constant that depends on $u_{c}$. Thus, to show that $f$ is a cusp form, it suffices to show that $\Phi\left(f\left[\mu_{c}\right]_{k}\right)=0$ for all $0<c \mid N$. Note that to do so, we need to consider the Siegel $\Phi$ map only on $\mathcal{M}_{k}(\mathrm{~K}(N))$. Recall that the Witt map on $\mathcal{M}_{k}(\mathrm{~K}(N))$ has codomain $\mathcal{M}_{k}\left(\mathrm{SL}_{2}(\mathbb{Z})\right) \otimes \mathcal{M}_{k}\left(\mathrm{SL}_{2}(\mathbb{Z})\right)\left[\left[\begin{array}{ll}N & 0 \\ 0 & 1\end{array}\right]\right]_{k}$.

If $k=2$ or $k$ is odd then $\mathcal{M}_{k}\left(\mathrm{SL}_{2}(\mathbb{Z})\right) \otimes \mathcal{M}_{k}\left(\mathrm{SL}_{2}(\mathbb{Z})\right)\left[\left[\begin{array}{cc}N & 0 \\ 0 & 1\end{array}\right]\right]_{k}=0$, making the Witt map on $\mathcal{M}_{k}(\mathrm{~K}(N))$ zero and hence making the Siegel $\Phi$ map on $\mathcal{M}_{k}(\mathrm{~K}(N))$ zero. This proves the first statement.

If $k=4,6,8,10,14$ then $\mathcal{M}_{k}\left(\mathrm{SL}_{2}(\mathbb{Z})\right) \otimes \mathcal{M}_{k}\left(\mathrm{SL}_{2}(\mathbb{Z})\right)\left[\left[\begin{array}{ll}N & 0 \\ 0 & 1\end{array}\right]\right]_{k}=\mathbb{C} \varphi$ with $\varphi(\tau, \omega)=$ $E_{k}(\tau) E_{k}\left[\left[\begin{array}{ll}N & 0 \\ 0 & 1\end{array}\right]\right]_{k}(\omega)$. The Witt map image of any $f$ in $\mathcal{M}_{k}(\mathrm{~K}(N))$ has the same constant term as $f$. Thus, letting $c_{o}$ denote the constant term of $E_{k}\left[\left[\begin{array}{cc}N & 0 \\ 0 & 1\end{array}\right]\right]_{k}$, the Witt map is $f \mapsto\left(a(0 ; f) / c_{o}\right) \varphi$ and Siegel's $\Phi$ map is $f \mapsto a(0 ; f) E_{k}$. To prove the nontrivial part of the second statement, we take any $f \in \mathcal{M}_{k}(\mathrm{~K}(N))$ with $a(0 ; f)=0$ and show that $\Phi\left(f\left[\mu_{c}\right]_{k}\right)=0$ for $0<c \mid N$, making $f$ a cusp form. The Fourier coefficients of $f\left[\mu_{c}\right]_{k}$ are $a\left(t ; f\left[\mu_{c}\right]_{k}\right)=a\left(t\left[\alpha_{c}^{\prime}\right] ; f\right)$, so in particular $a\left(0 ; f\left[\mu_{c}\right]_{k}\right)=a(0 ; f)=0$. Thus $\Phi\left(f\left[\mu_{c}\right]_{k}\right)=a\left(0 ; f\left[\mu_{c}\right]_{k}\right) E_{k}=0$, as desired. 


\section{Analyzing Weight 2 Via Weight 4}

Let $N$ be a positive integer. This section presents four tests to study $\mathcal{S}_{2}(\mathrm{~K}(N))$ based on computations in $\mathcal{S}_{4}(\mathrm{~K}(N))$. A main point is that the tests can certify that the results of Jacobi restriction are rigorous even when the restriction is carried out only to a few terms. We begin by introducing subspaces of $\mathcal{S}_{2}(\mathrm{~K}(N))$ whose vanishing connotes the correctness of Jacobi restriction.

Definition 4.1. For any $d \in \mathbb{Z}_{\geq 1}$ define

$$
\mathcal{S}_{2}(\mathrm{~K}(N))(d)=\left\{f \in \mathcal{S}_{2}(\mathrm{~K}(N)): f(\Omega)=\sum_{m \geq d} \phi_{m}(f)(\tau, z) \xi^{m N}\right\} .
$$

Also define

$$
\mathcal{S}_{2}(\mathrm{~K}(N))^{\epsilon}(d)=\mathcal{S}_{2}(\mathrm{~K}(N))(d) \cap \mathcal{S}_{2}(\mathrm{~K}(N))^{\epsilon}, \quad \epsilon= \pm 1 .
$$

We say that elements of a space $\mathcal{S}_{2}(\mathrm{~K}(N))(d)$ are $d$-docked, because their FourierJacobi coefficients before $\phi_{d}$ vanish; in particular, 1-docked connotes no conditions and 2-docked means that $\phi_{1}(f)=0$. Any $f \in \mathcal{S}_{2}(\mathrm{~K}(N))(d)$ has Fourier coefficients $a(t ; f)=0$ for all $t=\left[\begin{array}{cc}n & r / 2 \\ r / 2 & m N\end{array}\right] \in \mathcal{X}_{2}(N)$ such that $m<d$, and so it has Fourier coefficients $a(t[\gamma] ; f)=0$ for all such $t$ and for all $\gamma \in \Gamma_{ \pm}^{0}(N)$ because its Fourier coefficients are $\Gamma_{ \pm}^{0}(N)$ class functions, as discussed in section2 If $\mathcal{S}_{2}(\mathrm{~K}(N))(d)=0$, so that $\mathcal{S}_{2}(\mathrm{~K}(N))^{ \pm}(d)=0$, then running Jacobi restriction out to $d-1$ terms or more produces rigorous upper bounds of $\operatorname{dim} \mathcal{S}_{2}(\mathrm{~K}(N))^{ \pm}$. If $\mathcal{S}_{2}(\mathrm{~K}(N))^{+}(d)=0$ then running Jacobi restriction out to $d-1$ terms or more produces a rigorous upper bound of $\operatorname{dim} \mathcal{S}_{2}(\mathrm{~K}(N))^{+}$, and similarly with "-" in place of "+". Note, however, that the conditions $\mathcal{S}_{2}(\mathrm{~K}(N))^{ \pm}(d)=0$ need not imply $\mathcal{S}_{2}(\mathrm{~K}(N))(d)=0$ for $d \geq 2$. Note also that $\mathcal{S}_{2}(\mathrm{~K}(N))(d) \cap \operatorname{Grit}\left(\mathrm{J}_{2, N}^{\text {cusp }}\right)=0$ for $d \geq 2$. We make two observations to be used in the analysis.

Lemma 4.2. (a) Let $f \in \mathcal{S}_{2}(\mathrm{~K}(N))$ be nonzero, and let $\left\{g_{i}: i \in I\right\}$ be a basis of $\operatorname{Grit}\left(\mathrm{J}_{2, N}^{\text {cusp }}\right)$. The set $\left\{f g_{i}: i \in I\right\}$ is linearly independent in $\mathcal{S}_{4}(\mathrm{~K}(N))$, and if $f$ is a nonlift then the set $\left\{f^{2}, f g_{i}: i \in I\right\}$ is linearly independent as well.

(b) We have the equivalence

$$
\mathcal{S}_{2}(\mathrm{~K}(N))^{+}=\operatorname{Grit}\left(\mathrm{J}_{2, N}^{\text {cusp }}\right) \Longleftrightarrow \mathcal{S}_{2}(\mathrm{~K}(N))^{+}(2)=0,
$$

and the same equivalence holds with $\mathcal{S}_{2}(\mathrm{~K}(N))$ in place of $\mathcal{S}_{2}(\mathrm{~K}(N))^{+}$.

Proof. (a) Because there is no nontrivial linear relation among $\left\{g_{i}: i \in I\right\}$, there is no such relation among $\left\{f g_{i}: i \in I\right\}$ either, because the graded ring of paramodular forms has no zero divisors. The same argument shows that if $f$ is a nonlift then there is no nontrivial linear relation among $\left\{f^{2}, f g_{i}: i \in I\right\}$.

(b) A nonzero element of $\mathcal{S}_{2}(\mathrm{~K}(N))^{+}(2)$ is an element of $\mathcal{S}_{2}(\mathrm{~K}(N))^{+}$but not of $\operatorname{Grit}\left(\mathrm{J}_{2, N}^{\text {cusp }}\right)$, and an element of $\mathcal{S}_{2}(\mathrm{~K}(N))^{+}$that is not an element of $\operatorname{Grit}\left(\mathrm{J}_{2, N}^{\text {cusp }}\right)$ can be translated by an element of $\operatorname{Grit}\left(\mathrm{J}_{2, N}^{\text {cusp }}\right)$ to produce a nonzero element of $\mathcal{S}_{2}(\mathrm{~K}(N))^{+}(2)$. The argument for $\mathcal{S}_{2}(\mathrm{~K}(N))$ is the same.

To study $\mathcal{S}_{2}(\mathrm{~K}(N))(d)$ and $\mathcal{S}_{2}(\mathrm{~K}(N))^{\epsilon}(d)$ for $\epsilon= \pm 1$, we introduce subspaces of $\mathcal{S}_{4}(\mathrm{~K}(N))$ and $\mathcal{S}_{4}(\mathrm{~K}(N))^{\epsilon}$ that are generated by products of $\mathcal{S}_{2}(\mathrm{~K}(N))$-elements subject to docking and Fricke eigenspace conditions. 
Definition 4.3. For any $d \in \mathbb{Z}_{\geq 1}$, define the following subspaces of $\mathcal{S}_{4}(\mathrm{~K}(N))$.

$$
\begin{aligned}
& H_{4}(N, d, d)^{+}=\left\langle f_{1} f_{2}: f_{1}, f_{2} \in \mathcal{S}_{2}(\mathrm{~K}(N))^{\epsilon}(d) \text { for one of } \epsilon= \pm 1\right\rangle, \\
& H_{4}(N, d, 1)=\left\langle f_{1} f_{2}: f_{1} \in \mathcal{S}_{2}(\mathrm{~K}(N))(d), f_{2} \in \mathcal{S}_{2}(\mathrm{~K}(N))\right\rangle, \\
& H_{4}(N, d, 1)^{+}=\left\langle f_{1} f_{2}: f_{1} \in \mathcal{S}_{2}(\mathrm{~K}(N))^{\epsilon}(d), f_{2} \in \mathcal{S}_{2}(\mathrm{~K}(N))^{\epsilon} \text { for one of } \epsilon= \pm 1\right\rangle, \\
& H_{4}(N, d, 1)^{-}=\left\langle f_{1} f_{2}: f_{1} \in \mathcal{S}_{2}(\mathrm{~K}(N))^{\epsilon}(d), f_{2} \in \mathcal{S}_{2}(\mathrm{~K}(N))^{-\epsilon} \text { for one of } \epsilon= \pm 1\right\rangle .
\end{aligned}
$$

Dimension bounds of the weight 4 spaces $H_{4}(N)$ combine with Lemma 4.2 (a) to give information about the docked weight 2 spaces as follows.

Lemma 4.4. Let $d$ be a positive integer.

(1) If $H_{4}(N, d, d)^{+}=0$ then $\mathcal{S}_{2}(\mathrm{~K}(N))^{ \pm}(d)=0$.

(2) If $\operatorname{dim} H_{4}(N, d, 1)<\operatorname{dim} \mathrm{J}_{2, N}^{\text {cusp }}+1$ then $\mathcal{S}_{2}(\mathrm{~K}(N))(d) \subset \operatorname{Grit}\left(\mathrm{J}_{2, N}^{\text {cusp }}\right)$.

(3) If $\operatorname{dim} H_{4}(N, d, 1)^{+}<\operatorname{dim} \mathrm{J}_{2, N}^{\text {cusp }}+1$ then $\mathcal{S}_{2}(\mathrm{~K}(N))^{+}(d) \subset \operatorname{Grit}\left(\mathrm{J}_{2, N}^{\text {cusp }}\right)$.

(4) If $\operatorname{dim} H_{4}(N, d, 1)^{-}<\operatorname{dim} \mathrm{J}_{2, N}^{\text {cusp }}$ then $\mathcal{S}_{2}(\mathrm{~K}(N))^{-}(d)=0$.

Proof. (1) Every $f \in \mathcal{S}_{2}(\mathrm{~K}(N))^{\epsilon}(d)$ for either of $\epsilon= \pm 1$ squares into $H_{4}(N, d, d)^{+}$, which is 0 , so $\mathcal{S}_{2}(\mathrm{~K}(N))^{ \pm}(d)=0$.

(2) If some $f \in \mathcal{S}_{2}(\mathrm{~K}(N))(d)$ is a nonlift then the linear independence of $\left\{f^{2}, f g_{i}\right.$ : $i \in I\}$ gives $\operatorname{dim} H_{4}(N, d, 1) \geq \operatorname{dim} J_{2, N}^{\text {cusp }}+1$, and so the result follows by contraposition.

This same argument, but with plus spaces, gives (3).

(4) If some $f \in \mathcal{S}_{2}(\mathrm{~K}(N))^{-}(d)$ is nonzero then the linear independence of $\left\{f g_{i}: i \in I\right\}$ gives $\operatorname{dim} H_{4}(N, d, 1)^{-} \geq \operatorname{dim} \mathrm{J}_{2, N}^{\text {cusp }}$, and so the result follows by contraposition.

A crucial idea is that we can establish computable dimension estimates for the $H_{4}(N)$ spaces of Definition 4.3. The Fourier coefficient formula for the product of two weight 2 paramodular forms $f_{1}, f_{2} \in \mathcal{S}_{2}(\mathrm{~K}(N))$ is

$$
a\left(t ; f_{1} f_{2}\right)=\sum_{\substack{t_{1}, t_{2} \in \mathcal{X}_{2}(N) \\ t_{1}+t_{2}=t}} a\left(t_{1} ; f_{1}\right) a\left(t_{2} ; f_{2}\right), \quad t \in \mathcal{X}_{2}(N) .
$$

Recall that the Fourier coefficients of $f_{1}$ and $f_{2}$ are $\Gamma_{ \pm}^{0}(N)$ class functions. For any $t \in \mathcal{X}_{2}(N)$, let $m_{N}(t)=\min \left\{m:\left[\begin{array}{cc}n & r / 2 \\ r / 2 & m N\end{array}\right] \in t\left[\Gamma_{ \pm}^{0}(N)\right]\right\}$. (The " $m$ " in this function's name stands for "minimum function," although also the quantity being minimized is named $m$.) Computing $m_{N}(t)$ is a finite process, because for any given $m$ we may take $|r| \leq m N$, then search for matrices $\left[\begin{array}{cc}n & r / 2 \\ r / 2 & m N\end{array}\right]$ such that $4 n m N-r^{2}=4 \operatorname{det} t$, and then check whether each such matrix lies in $t\left[\Gamma_{ \pm}^{0}(N)\right]$. Consider an index $t \in \mathcal{X}_{2}(N)$, and let $d$ be a positive integer. Suppose that for any pair $t_{1} \times t_{2}$ of $\mathcal{X}_{2}(N)$ matrices such that $t_{1}+t_{2}=t$, necessarily $m_{N}\left(t_{1}\right)<d$ or $m_{N}\left(t_{2}\right)<d$. This condition combines with the previous display to say that $a\left(t ; f_{1} f_{2}\right)=0$ for every generating product $f_{1} f_{2}$ of $H_{4}(N, d, d)^{+}$, and consequently $a(t ; f)=0$ for all $f \in H_{4}(N, d, d)^{+}$. Similarly, if for any pair $t_{1} \times t_{2}$ such that $t_{1}+t_{2}=t$, necessarily $m_{N}\left(t_{1}\right)<d$, then $a(t ; f)=0$ for all $f \in H_{4}(N, d, 1)$.

In the next proposition, typewriter font is used to denote variables that we compute in practice. We remind the reader that $\operatorname{dim} \mathcal{S}_{4}(\mathrm{~K}(N))$ and $\operatorname{dim} \mathrm{J}_{2, N}^{\text {cusp }}$ are known [IK, EZ85, SZ89, but not currently $\operatorname{dim} \mathcal{S}_{4}(\mathrm{~K}(N))^{ \pm}$. 
Proposition 4.5. Let $N$ be a positive integer. Let $\mathrm{S}^{ \pm}$be subspaces of $\mathcal{S}_{4}(\mathrm{~K}(N))^{ \pm}$, and let $\mathrm{S}=\mathrm{S}^{+} \oplus \mathrm{S}^{-} \subset \mathcal{S}_{4}(\mathrm{~K}(N))$. For each $s$ in $\{+,-$, empty character $\}$, let $\left\{g_{i}^{S}\right\}$ be a basis of $\mathrm{S}^{s}$. Let $d$ be a positive integer. For each $\delta$ in $\{d, 1\}$ and each $s$ as above, let $\mathcal{T}\left(\mathrm{S}^{s}\right)$ be a determining set of Fourier coefficient indices for $\mathrm{S}^{s}$, and let

$$
\mathcal{T}\left(\mathbf{S}^{s}, d, \delta\right)=\left\{t \in \mathcal{T}\left(\mathrm{S}^{s}\right):\left(\begin{array}{l}
\text { if } t=t_{1}+t_{2} \text { where } t_{1}, t_{2} \in \mathcal{X}_{2}(N) \\
\text { then } m_{N}\left(t_{1}\right)<d \text { or } m_{N}\left(t_{2}\right)<\delta
\end{array}\right)\right\} .
$$

(For $\delta=1$, the condition $m_{N}\left(t_{2}\right)<\delta$ is impossible, leaving a condition on $t_{1}$.) Define the $\operatorname{dim} \mathrm{S}^{s} \times\left|\mathcal{T}\left(\mathrm{S}^{s}, d, \delta\right)\right|$ matrix $\mathrm{M}\left(\mathrm{S}^{s}, d, \delta\right)=\left[a\left(t ; g_{i}^{s}\right)\right]$. We have the following bounds.

(1) $\operatorname{dim} H_{4}(N, d, d)^{+} \leq \operatorname{dim} \mathcal{S}_{4}(\mathrm{~K}(N))-\operatorname{dim} \mathrm{S}^{-}-\operatorname{rank} \mathrm{M}\left(\mathrm{S}^{+}, d, d\right)$,

(2) $\operatorname{dim} H_{4}(N, d, 1) \leq \operatorname{dim} \mathcal{S}_{4}(\mathrm{~K}(N))-\operatorname{rank} \mathrm{M}(\mathrm{S}, d, 1)$,

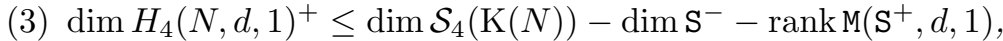

(4) $\operatorname{dim} H_{4}(N, d, 1)^{-} \leq \operatorname{dim} \mathcal{S}_{4}(\mathrm{~K}(N))-\operatorname{dim} \mathrm{S}^{+}-\operatorname{rank} \mathrm{M}\left(\mathrm{S}^{-}, d, 1\right)$.

Proof. We prove the first bound. An element of $H_{4}(N, d, d)^{+}$either lies outside $\mathrm{S}^{+}$ or it lies in $\mathrm{S}^{+}$and its Fourier series expansion truncation is 0 on $\mathcal{T}(N, d, d)$. Thus $\operatorname{dim} H_{4}(N, d, d)^{+}$is at most the sum of $\operatorname{dim} \mathcal{S}_{4}(\mathrm{~K}(N))^{+}-\operatorname{dim} \mathrm{S}^{+}$and the left nullity of $\mathrm{M}\left(\mathrm{S}^{+}, d, d\right)$. But $\operatorname{dim} \mathcal{S}_{4}(\mathrm{~K}(N))^{+} \leq \operatorname{dim} \mathcal{S}_{4}(\mathrm{~K}(N))-\operatorname{dim} \mathrm{S}^{-}$and the left nullity is $\operatorname{dim} \mathrm{S}^{+}-\operatorname{rank} \mathrm{M}\left(\mathrm{S}^{+}, d, d\right)$, so the stated bound follows. The other three bounds are established similarly.

In practice we grow our spanned subspaces $\mathrm{S}^{ \pm}$of $\mathcal{S}_{4}(\mathrm{~K}(N))^{ \pm}$until $\operatorname{dim} \mathrm{S}=$ $\operatorname{dim} \mathcal{S}_{4}(\mathrm{~K}(N))$ if $\mathrm{J}_{2, N}^{\text {cusp }}=0$, or $\operatorname{dim} \mathcal{S}_{4}(\mathrm{~K}(N))-\operatorname{dim} \mathrm{S}<\operatorname{dim} \mathrm{J}_{2, N}^{\text {cusp }}$ if $\mathrm{J}_{2, N}^{\text {cusp }} \neq 0$. As $d$ grows, we expect $\operatorname{rank} \mathrm{M}\left(\mathrm{S}^{s}, d, \delta\right)$ to grow toward $\operatorname{dim} \mathrm{S}^{s}$, making all four bounds in Proposition 4.5 decrease toward $\operatorname{dim} \mathcal{S}_{4}(\mathrm{~K}(N))-\operatorname{dim} \mathrm{S}$, and so we expect the conditions of Lemma 4.4 to apply for large enough $d$. Building on Lemma 4.4 and Proposition 4.5, we have diagnostic tests for the weight 2 spaces as follows.

Proposition 4.6. Let $\mathrm{S}^{ \pm}$and $\mathrm{S}$ be as in Proposition 4.5. Let d be a positive integer, and let $\mathrm{M}\left(\mathrm{S}^{+}, d, d\right), \mathrm{M}(\mathrm{S}, d, 1)$, and $\mathrm{M}\left(\mathrm{S}^{ \pm}, d, 1\right)$ be as in Proposition 4.5 .

(1) Suppose that $\operatorname{dim} \mathcal{S}_{4}(\mathrm{~K}(N))=\operatorname{dim} \mathrm{S}^{-}+\operatorname{rank} \mathrm{M}\left(\mathrm{S}^{+}, d, d\right)$. If $d=1$ then $\mathcal{S}_{2}(\mathrm{~K}(N))=0$; if $d=2$ then $\mathcal{S}_{2}(\mathrm{~K}(N))^{+}=\operatorname{Grit}\left(\mathrm{J}_{2, N}^{\text {cusp }}\right)$ and $\mathcal{S}_{2}(\mathrm{~K}(N))^{-}(2)=$ 0 ; if $d \geq 3$ then $\mathcal{S}_{2}(\mathrm{~K}(N))^{ \pm}(d)=0$.

(2) Suppose that $\operatorname{dim} \mathcal{S}_{4}(\mathrm{~K}(N))-\operatorname{rank} \mathrm{M}(\mathrm{S}, d, 1)<\operatorname{dim} \mathrm{J}_{2, N}^{\text {cusp }}+1$. If $d=1,2$ then $\mathcal{S}_{2}(\mathrm{~K}(N))=\operatorname{Grit}\left(\mathrm{J}_{2, N}^{\text {cusp }}\right)$; if $d \geq 3$ then $\mathcal{S}_{2}(\mathrm{~K}(N))^{ \pm}(d)=0$.

(3) Suppose that $\operatorname{dim} \mathcal{S}_{4}(\mathrm{~K}(N))-\operatorname{dim} \mathrm{S}^{-}-\operatorname{rank} \mathrm{M}\left(\mathrm{S}^{+}, d, 1\right)<\operatorname{dim} \mathrm{J}_{2, N}^{\text {cusp }}+1$. If $d=1,2$ then $\mathcal{S}_{2}(\mathrm{~K}(N))^{+}=\operatorname{Grit}\left(\mathrm{J}_{2, N}^{\text {cusp }}\right)$; if $d \geq 3$ then $\mathcal{S}_{2}(\mathrm{~K}(N))^{+}(d)=0$.

(4) Suppose that $\operatorname{dim} \mathcal{S}_{4}(\mathrm{~K}(N))-\operatorname{dim} \mathrm{S}^{+}-\operatorname{rank} \mathrm{M}\left(\mathrm{S}^{-}, d, 1\right)<\operatorname{dim} J_{2, N}^{\text {cusp }}$. If $d=1$ then $\mathcal{S}_{2}(\mathrm{~K}(N))^{-}=0$. If $d \geq 2$ then $\mathcal{S}_{2}(\mathrm{~K}(N))^{-}(d)=0$.

We reiterate that the conclusion $\mathcal{S}_{2}(\mathrm{~K}(N))^{-}(2)=0$ in the first case implies that Jacobi restriction to one or more terms produces a rigorous upper bound of $\operatorname{dim} \mathcal{S}_{2}(\mathrm{~K}(N))^{-}$, and similarly for the other cases.

Before proving the proposition, we note that in the first case, the condition can hold only if $\operatorname{dim} \mathrm{S}=\operatorname{dim} \mathcal{S}_{4}(\mathrm{~K}(N))$, so this equality should be checked before computing $\operatorname{rank} \mathrm{M}\left(\mathrm{S}^{+}, d, d\right)$, and when this equality does hold, the condition simplifies to rank $\mathrm{M}\left(\mathrm{S}^{+}, d, d\right)=\operatorname{dim} \mathrm{S}^{+}$. Similarly, in the second and third cases, the condition can hold only if $\operatorname{dim} \mathcal{S}_{4}(\mathrm{~K}(N))-\operatorname{dim} \mathrm{S}<\operatorname{dim} \mathrm{J}_{2, N}^{\text {cusp }}+1$, and this inequality should 
be checked before computing $\operatorname{rank} \mathrm{M}(\mathrm{S}, d, 1)$ or $\operatorname{rank} \mathrm{M}\left(\mathrm{S}^{+}, d, 1\right)$. In the fourth case, the condition can hold only if $\operatorname{dim} \mathcal{S}_{4}(\mathrm{~K}(N))-\operatorname{dim} \mathrm{S}<\operatorname{dim} \mathrm{J}_{2, N}^{\text {cusp }}$, and this should be checked before computing $\operatorname{rank} \mathrm{M}\left(\mathrm{S}^{-}, d, 1\right)$.

Proof. For (1), by Lemma 4.4 and Proposition 4.5, $\mathcal{S}_{2}(\mathrm{~K}(N))^{ \pm}(d)=0$. For $d=1$ these equalities give $\mathcal{S}_{2}(\mathrm{~K}(N))=0$, and for $d=2$ the first equality implies that $\mathcal{S}_{2}(\mathrm{~K}(N))^{+}=\operatorname{Grit}\left(\mathrm{J}_{2, N}^{\mathrm{cusp}}\right)$ by Lemma $4.2(\mathrm{~b})$.

For (2), by Lemma 4.4 and Proposition 4.5. $\mathcal{S}_{2}(\mathrm{~K}(N))(d) \subset \operatorname{Grit}\left(\mathrm{J}_{2, N}^{\text {cusp }}\right)$. For $d=1$ this containment gives $\mathcal{S}_{2}(\mathrm{~K}(N))=\operatorname{Grit}\left(\mathrm{J}_{2, N}^{\mathrm{cusp}}\right)$. For $d=2$ it says that $\mathcal{S}_{2}(\mathrm{~K}(N))(2)=0$ because $\mathcal{S}_{2}(\mathrm{~K}(N))(2) \cap \operatorname{Grit}\left(\mathrm{J}_{2, N}^{\text {cusp }}\right)=0$, and so $\mathcal{S}_{2}(\mathrm{~K}(N))=$ $\operatorname{Grit}\left(\mathrm{J}_{2, N}^{\text {cusp }}\right)$ by Lemma $4.2(\mathrm{~b})$. For $d \geq 3$ it says that $\mathcal{S}_{2}(\mathrm{~K}(N))(d)=0$ because $\mathcal{S}_{2}(\mathrm{~K}(N))(d) \cap \operatorname{Grit}\left(\mathrm{J}_{2, N}^{\text {cusp }}\right)=0$.

The same argument, but with plus spaces, gives (3).

For (4), by Lemma 4.4 and Proposition 4.5, $\mathcal{S}_{2}(\mathrm{~K}(N))^{-}(d)=0$.

We have run Jacobi restriction to five or more terms for all of the spaces $\mathcal{S}_{2}(\mathrm{~K}(N))^{ \pm}$where the level $N$ is composite and squarefree in $\{62, \ldots, 299\}$. If one of the tests above applies for some $d \leq 6$ then the corresponding dimension upper estimate provided by Jacobi restriction to five or more terms is rigorous. The heuristic upper bounds provided by Jacobi restriction for the just-mentioned levels $N$ are

$$
\begin{array}{ll}
\operatorname{dim} \mathcal{S}_{2}(\mathrm{~K}(N))^{+} \leq \operatorname{dim} \mathrm{J}_{2, N}^{\text {cusp }} & \text { for } N \neq 249,295, \\
\operatorname{dim} \mathcal{S}_{2}(\mathrm{~K}(N))^{+} \leq \operatorname{dim} \mathrm{J}_{2, N}^{\text {cusp }}+1 & \text { for } N=249,295, \\
\operatorname{dim} \mathcal{S}_{2}(\mathrm{~K}(N))^{-}=0 & \text { for all } N .
\end{array}
$$

Thus our tests to certify that Jacobi restriction to five terms gives rigorous upper bounds are as follows.

$\mathbf{H}_{\mathbf{4}}(\mathbf{N}, \mathbf{d}, \mathbf{d})^{+}$test, can succeed only if $\operatorname{dim} \mathrm{S}=\operatorname{dim} \mathcal{S}_{4}(\mathrm{~K}(N))$ : For $d=1, \ldots, 6$, if $\operatorname{rank} \mathrm{M}\left(\mathrm{S}^{+}, d, d\right)=\operatorname{dim} \mathrm{S}^{+}$then

$$
\begin{array}{ll}
\mathcal{S}_{2}(\mathrm{~K}(N))=\operatorname{Grit}\left(\mathrm{J}_{2, N}^{\text {cusp }}\right) & \text { if } d=1 \text { or } 2, \text { or } d \geq 3 \text { and } N \neq 249,295, \\
\left(\begin{array}{l}
\operatorname{dim} \mathcal{S}_{2}(\mathrm{~K}(N))^{+} \leq \operatorname{dim} \mathrm{J}_{2, N}^{\text {cusp }}+1 \\
\text { and } \mathcal{S}_{2}(\mathrm{~K}(N))^{-}=0
\end{array}\right) \quad \text { if } d \geq 3 \text { and } N=249,295 .
\end{array}
$$

For $d=1$ this test can conclude that $\mathcal{S}_{2}(\mathrm{~K}(N))=0$, but the given conclusion is all that we need.

$\mathbf{H}_{\mathbf{4}}(\mathbf{N}, \mathbf{d}, \mathbf{1})$ test, can succeed only if $\operatorname{dim} \mathcal{S}_{4}(\mathrm{~K}(N))-\operatorname{dim} \mathrm{S}<\operatorname{dim} \mathrm{J}_{2, N}^{\text {cusp }}+1$ : For $d=1, \ldots, 6$, if $\operatorname{dim} \mathcal{S}_{4}(\mathrm{~K}(N))-\operatorname{rank} \mathrm{M}(\mathrm{S}, d, 1)<\operatorname{dim} \mathrm{J}_{2, N}^{\text {cusp }}+1$ then

$$
\begin{array}{ll}
\mathcal{S}_{2}(\mathrm{~K}(N))=\operatorname{Grit}\left(\mathrm{J}_{2, N}^{\text {cusp }}\right) & \text { if } d=1 \text { or } 2, \text { or } d \geq 3 \text { and } N \neq 249,295, \\
\left(\begin{array}{l}
\operatorname{dim} \mathcal{S}_{2}(\mathrm{~K}(N))^{+} \leq \operatorname{dim} \mathrm{J}_{2, N}^{\text {cusp }}+1 \\
\text { and } \mathcal{S}_{2}(\mathrm{~K}(N))^{-}=0
\end{array}\right) \quad \text { if } d \geq 3 \text { and } N=249,295 .
\end{array}
$$

$\mathbf{H}_{\mathbf{4}}(\mathbf{N}, \mathbf{d}, \mathbf{1})^{+}$test, can succeed only if $\operatorname{dim} \mathcal{S}_{4}(\mathrm{~K}(N))-\operatorname{dim} \mathrm{S}<\operatorname{dim} \mathrm{J}_{2, N}^{\text {cusp }}+1$ : For $d=1, \ldots, 6$, if $\operatorname{dim} \mathcal{S}_{4}(\mathrm{~K}(N))-\operatorname{dim} \mathrm{S}^{-}-\operatorname{rank} \mathrm{M}\left(\mathrm{S}^{+}, d, 1\right)<\operatorname{dim} \mathrm{J}_{2, N}^{\text {cusp }}+1$ then

$$
\begin{array}{ll}
\mathcal{S}_{2}(\mathrm{~K}(N))^{+}=\operatorname{Grit}\left(\mathrm{J}_{2, N}^{\text {cusp }}\right) & \text { if } d=1 \text { or } 2, \text { or } d \geq 3 \text { and } N \neq 249,295, \\
\operatorname{dim} \mathcal{S}_{2}(\mathrm{~K}(N))^{+} \leq \operatorname{dim} \mathrm{J}_{2, N}^{\text {cusp }}+1 & \text { if } d \geq 3 \text { and } N=249,295 .
\end{array}
$$


$\mathbf{H}_{\mathbf{4}}(\mathbf{N}, \mathbf{d}, \mathbf{1})^{-}$test, can succeed only if $\operatorname{dim} \mathcal{S}_{4}(\mathrm{~K}(N))-\operatorname{dim} \mathrm{S}<\operatorname{dim} \mathrm{J}_{2, N}^{\text {cusp }}:$ For $d=1, \ldots, 6$, if $\operatorname{dim} \mathcal{S}_{4}(\mathrm{~K}(N))-\operatorname{dim} \mathrm{S}^{+}-\operatorname{rank} \mathrm{M}\left(\mathrm{S}^{-}, d, 1\right)<\operatorname{dim} J_{2, N}^{\text {cusp }}$ then

$$
\mathcal{S}_{2}(\mathrm{~K}(N))^{-}=0 \text {. }
$$

\section{Tracing Down}

Let $N$ be a squarefree positive integer, and let $q$ be a prime that does not divide $N$. We define and compute an averaging trace down operator

$$
\operatorname{TrDn}: \mathcal{S}_{k}(\mathrm{~K}(N q)) \longrightarrow \mathcal{S}_{k}(\mathrm{~K}(N)) .
$$

Here $\mathrm{K}(N q)$ is not a subgroup of $\mathrm{K}(N)$, but we have the configuration of groups (in which $\Gamma_{0}^{\prime}(N)$ denotes $\mathrm{K}(N) \cap \mathrm{Sp}_{2}(\mathbb{Z})$ and similarly for $\Gamma_{0}^{\prime}(N q)$ )

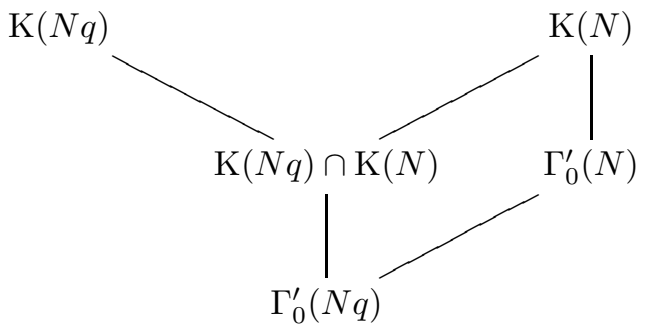

We will find representatives $\left\{g_{1 i}\right\}$ of the quotient space $\Gamma_{0}^{\prime}(N q) \backslash \Gamma_{0}^{\prime}(N)$ and representatives $\left\{g_{2 j}\right\}$ of the quotient space $\Gamma_{0}^{\prime}(N) \backslash \mathrm{K}(N)$, so that altogether $\mathrm{K}(N)=$ $\bigsqcup_{i, j} \Gamma_{0}^{\prime}(N q) g_{i j}$ with $g_{i j}=g_{1 i} g_{2 j}$ for all $i, j$. Further, because $N q$ is squarefree, so that $\mathrm{K}(N q)$ has only one 0 -cusp, we can decompose each coset representative $g=g_{i j}$ as $g=\kappa u$ with $\kappa \in \mathrm{K}(N q)$ and $u \in \mathrm{P}_{2,0}(\mathbb{Q})$. Thus overall the quotient space $\Gamma_{0}^{\prime}(N q) \backslash \mathrm{K}(N)$ is $\mathrm{K}(N)=\bigsqcup \Gamma_{0}^{\prime}(N q) \kappa u$, and because each $\kappa$ lies in $\mathrm{K}(N q)$ the trace down operator is

$$
\operatorname{TrDn} f=\sum_{u} f[u]_{k}, \quad f \in \mathcal{S}_{k}(\mathrm{~K}(N q)) .
$$

Some of the results in this section are well known, but we assemble them here for the sake of a complete discussion in one place.

First we study $\Gamma_{0}^{\prime}(N q) \backslash \Gamma_{0}^{\prime}(N)$. Let $\mathbb{P}^{3}(\mathbb{Z} / q \mathbb{Z})=(\mathbb{Z} / q \mathbb{Z})_{\text {prim }}^{4} /(\mathbb{Z} / q \mathbb{Z})^{\times}$where $(\mathbb{Z} / q \mathbb{Z})_{\text {prim }}^{4}$ consists of all vectors $(\bar{a}, \bar{b}, \bar{c}, \bar{d}) \in(\mathbb{Z} / q \mathbb{Z})^{4}$ such that the ideal of $\mathbb{Z} / q \mathbb{Z}$ generated by the entries $\bar{a}, \bar{b}, \bar{c}, \bar{d}$ is all of $\mathbb{Z} / q \mathbb{Z}$; here the overbar denotes reduction of integers modulo $q \mathbb{Z}$. We show that $\mathbb{P}^{3}(\mathbb{Z} / q \mathbb{Z})$ parametrizes $\Gamma_{0}^{\prime}(N q) \backslash \Gamma_{0}^{\prime}(N)$.

Proposition 5.1. Let $N$ be a positive integer and let $q$ be a prime that does not divide $N$.

(1) Each element $\pi$ of $\mathbb{P}^{3}(\mathbb{Z} / q \mathbb{Z})$ has a representative $(a, b, c, d) \in \mathbb{Z}^{4}$ such that the vector $v_{\pi}=(a N, b N, c N, d)$ is primitive, i.e., $\operatorname{gcd}(a N, b N, c N, d)=1$.

(2) Each such $v_{\pi}$ is the bottom row of a matrix $g_{\pi}$ in $\Gamma_{0}^{\prime}(N)$.

(3) The map $\mathbb{P}^{3}(\mathbb{Z} / q \mathbb{Z}) \longrightarrow \Gamma_{0}^{\prime}(N q) \backslash \Gamma_{0}^{\prime}(N)$ that takes $\pi$ to $\Gamma_{0}^{\prime}(N q) g_{\pi}$ is well defined. That is, the coset $\Gamma_{0}^{\prime}(N q) g_{\pi}$ depends only on $\pi$, not on any choices made in constructing $v_{\pi}$ from $\pi$ or $g_{\pi}$ from $v_{\pi}$.

(4) The map is bijective. That is, if $\pi$ and $\pi^{\prime}$ are distinct in $\mathbb{P}^{3}(\mathbb{Z} / q \mathbb{Z})$ then $\Gamma_{0}^{\prime}(N) g_{\pi}$ and $\Gamma_{0}^{\prime}(N) g_{\pi^{\prime}}$ are distinct in $\Gamma_{0}^{\prime}(N q) \backslash \Gamma_{0}^{\prime}(N)$, and cosets $\Gamma_{0}^{\prime}(N q) g_{\pi}$ constitute all of $\Gamma_{0}^{\prime}(N q) \backslash \Gamma_{0}^{\prime}(N)$. Thus $\left[\Gamma_{0}^{\prime}(N): \Gamma_{0}^{\prime}(N q)\right]=\left|\mathbb{P}^{3}(\mathbb{Z} / q \mathbb{Z})\right|=$ $1+q+q^{2}+q^{3}$. 
Proof. (1) Consider any element $\pi=(\bar{\alpha}, \bar{\beta}, \bar{\gamma}, \bar{\delta})(\mathbb{Z} / q \mathbb{Z})^{\times}$of $\mathbb{P}^{3}(\mathbb{Z} / q \mathbb{Z})$. Take a representative $(\alpha, \beta, \gamma, \delta+m q)$ such that $\operatorname{gcd}(\delta+m q, N)=1$, and then divide through by the greatest common divisor of the entries; because $q$ cannot divide all four entries, this has no effect on the element $\pi$ represented. We now have the desired representative $(a, b, c, d)$ of $\pi$ such that $\operatorname{gcd}(a N, b N, c N, d)=1$.

(2) Because the first three entries of $v_{\pi}$ are multiples of $N$, and because any $\mathrm{Sp}_{2}(\mathbb{Z})$ matrix with such a bottom row lies in $\Gamma_{0}^{\prime}(N)$, it suffices to review the standard fact that any primitive vector $r_{4} \in \mathbb{Z}^{4}$ is the bottom row of a matrix in $\operatorname{Sp}_{2}(\mathbb{Z})$. There exists $r_{2} \in \mathbb{Z}^{4}$ such that $r_{2} J r_{4}^{\prime}=-1$. Take a primitive $r_{3}$ such that $r_{3} J r_{2}^{\prime}=r_{3} J r_{4}^{\prime}=0$, and then take $\rho_{1}$ such that $\rho_{1} J r_{3}^{\prime}=-1$. Let $r_{1}=\rho_{1}+\left(\rho_{1} J r_{4}^{\prime}\right) r_{2}-\left(\rho_{1} J r_{2}^{\prime}\right) r_{4}$. Thus $r_{1} J r_{3}^{\prime}=-1$ and $r_{1} J r_{2}^{\prime}=r_{1} J r_{4}^{\prime}=0$. The matrix with rows $r_{1}$ through $r_{4}$ lies in $\operatorname{Sp}_{2}(\mathbb{Z})$.

(3) Consider any two $\Gamma_{0}^{\prime}(N)$ matrices $g_{\pi}$ and $\tilde{g}_{\pi}$ arising from the same element $\pi$ of $\mathbb{P}^{3}(\mathbb{Z} / q \mathbb{Z})$. We want to show that $\tilde{g}_{\pi} g_{\pi}^{-1}$ lies in $\Gamma_{0}^{\prime}(N q)$. Because $\Gamma_{0}^{\prime}(N)$ is a group, all that needs to be shown is that the first three entries of the bottom row of $\tilde{g}_{\pi} g_{\pi}^{-1}$ are multiples of $q$. The matrices $g_{\pi}$ and $\tilde{g}_{\pi}$ have bottom rows $r_{4}=(\alpha N, \beta N, \gamma N, \delta)$ and $\tilde{r}_{4}=(\tilde{\alpha} N, \tilde{\beta} N, \tilde{\gamma} N, \tilde{\delta})$ with $(\tilde{\alpha}, \tilde{\beta}, \tilde{\gamma}, \tilde{\delta})=\lambda(\alpha, \beta, \gamma, \delta) \bmod q$ for some $\lambda$ coprime to $q$, and so working modulo $q$ we may replace the bottom row $\tilde{r}_{4}$ of $\tilde{g}_{\pi}$ by $\lambda r_{4}$, a scalar multiple of the bottom row of $g_{\pi}$. Thus the bottom row of $\tilde{g}_{\pi} g_{\pi}^{-1}$ is a multiple modulo $q$ of the bottom row $(0,0,0,1)$ of $g_{\pi} g_{\pi}^{-1}=1_{4}$, and we are done.

(4) For injectivity, consider any $\pi \in \mathbb{P}^{3}(\mathbb{Z} / q \mathbb{Z})$ and consider a matrix $g_{\pi} \in$ $\Gamma_{0}^{\prime}(N)$, whose bottom row is $(\alpha N, \beta N, \gamma N, \delta)$ where $\pi=(\bar{\alpha}, \bar{\beta}, \bar{\gamma}, \bar{\delta})(\mathbb{Z} / q \mathbb{Z})^{\times}$. Any $h \in \Gamma_{0}^{\prime}(N q)$ has bottom row $(a N q, b N q, c N q, d)$, with $q \nmid d$, and so the product $h g_{\pi}$ has bottom row $(d \alpha N+* N q, d \beta N+* N q, d \gamma N+* N q, d \delta+* N q)$, which is $((d \alpha+* q) N,(d \beta+* q) N,(d \gamma+* q) N, d \delta+* q)$. This shows that the coset $\Gamma_{0}^{\prime}(N q) g_{\pi}$ consists entirely of matrices $g_{\pi^{\prime}}$. For surjectivity, any element $g$ of $\Gamma_{0}^{\prime}(N)$ has bottom row $(a N, b N, c N, d)$ with the vector primitive. Thus $(a, b, c, d)$ is primitive as well, and so it represents an element $\pi$ of $\mathbb{P}^{3}(\mathbb{Z} / q \mathbb{Z})$, and $g$ takes the form $g=g_{\pi}$. The index $\left[\Gamma_{0}^{\prime}(N): \Gamma_{0}^{\prime}(N q)\right]=\left|\mathbb{P}^{3}(\mathbb{Z} / q \mathbb{Z})\right|$ follows from the bijectivity.

Next we study $\Gamma_{0}^{\prime}(N) \backslash \mathrm{K}(N)$. Let $\Gamma^{0}(N)$ denote the subgroup of $\mathrm{SL}_{2}(\mathbb{Z})$ defined by the condition $b=0 \bmod N$. We show that $\Gamma^{0}(N) \backslash \mathrm{SL}_{2}(\mathbb{Z})$ parametrizes $\Gamma_{0}^{\prime}(N) \backslash \mathrm{K}(N)$.

Proposition 5.2. For each matrix $g_{o}=\left[\begin{array}{ll}a & b \\ c & d\end{array}\right]$ of $\mathrm{SL}_{2}(\mathbb{Z})$, define a corresponding matrix

$$
g=\iota\left(1_{2},\left[\begin{array}{ll}
1 & 0 \\
0 & N
\end{array}\right] g_{o}\left[\begin{array}{cc}
1 & 0 \\
0 & 1
\end{array} / N\right]\right)=\left[\begin{array}{cc|cc}
1 & 0 & 0 & 0 \\
0 & a & 0 & b / N \\
\hline 0 & 0 & 1 & 0 \\
0 & c N & 0 & d
\end{array}\right] .
$$

Then $g$ lies in $\mathrm{K}(N)$, and the map $\Gamma^{0}(N) g_{o} \mapsto \Gamma_{0}^{\prime}(N)$ from $\Gamma^{0}(N) \backslash \mathrm{SL}_{2}(\mathbb{Z})$ to $\Gamma_{0}^{\prime}(N) \backslash \mathrm{K}(N)$ is well defined and bijective. Thus $\left[\mathrm{K}(N): \Gamma_{0}^{\prime}(N)\right]=\left[\mathrm{SL}_{2}(\mathbb{Z})\right.$ : $\left.\Gamma^{0}(N)\right]=N \prod_{p \mid N}(1+1 / p)$.

Proof. One readily checks that $g \in \mathrm{K}(N)$ and that the map $\Gamma^{0}(N) g_{o} \mapsto \Gamma_{0}^{\prime}(N) g$ is well defined and injective. For surjectivity, consider any element $h$ of $\mathrm{K}(N)$ that does not lie in $\Gamma_{0}^{\prime}(N)$, and let $h^{-1}$ have last column $\left(*, \beta_{o} / N, *, \delta_{o}\right)^{\prime}$. Note that $\beta_{o} \neq$ 0 , because otherwise $h^{-1}$ lies in $\Gamma_{0}^{\prime}(N)$ and hence so does $h$. Let $\epsilon=\operatorname{gcd}\left(\beta_{o}, \delta_{o}\right)$. There is an element $h_{o}=\left[\begin{array}{ll}\alpha_{o} & \beta_{o} / \epsilon \\ \gamma_{o} & \delta_{o} / \epsilon\end{array}\right]$ of $\mathrm{SL}_{2}(\mathbb{Z})$; let $g_{o}=h_{o}^{-1}=\left[\begin{array}{cc}\delta_{o} / \epsilon & -\beta_{o} / \epsilon \\ -\gamma_{o} & \alpha_{o}\end{array}\right]$, and 
let $g$ correspond to $g_{o}$ as above. The $(2,4)$-entry of $g h^{-1}$ is the inner product of $\left(0, \delta_{o} / \epsilon, 0,-\beta_{o} /(\epsilon N)\right)$ and $\left(*, \beta_{o} / N, *, \delta_{o}\right)$, which is 0 . Thus $g h^{-1} \in \mathrm{K}(N) \cap$ $\operatorname{Sp}_{2}(\mathbb{Z})=\Gamma_{0}^{\prime}(N)$ and so $\Gamma_{0}^{\prime}(N) h=\Gamma_{0}^{\prime}(N) g$.

Third, we study upper triangular representatives. Theorem 1.4 of [PY13] (see also Ibu93]) specializes to show for squarefree $N q$ that $\mathrm{Sp}_{2}(\mathbb{Q})=\mathrm{K}(N q) \mathrm{P}_{2,0}(\mathbb{Q})$ where $\mathrm{P}_{2,0}(\mathbb{Q})$ is the Siegel parabolic group of rational symplectic matrices having $c$-block 0. Furthermore, the proof of the theorem can be made algorithmic, and doing so is especially easy for squarefree $N$. With representatives $\left\{g_{1 i}\right\}$ and $\left\{g_{2 j}\right\}$ of $\Gamma_{0}^{\prime}(N q) \backslash \Gamma_{0}^{\prime}(N)$ of $\Gamma_{0}^{\prime}(N) \backslash \mathrm{K}(N)$ at hand, we thus have an algorithm to decompose the representatives $\left\{g_{i j}\right\}=\left\{g_{1, i} g_{2, j}\right\}$ of $\Gamma_{0}^{\prime}(N q) \backslash \mathrm{K}(N)$ as $\left\{\kappa_{i j} u_{i j}\right\}$ with each $\kappa_{i j} \in \mathrm{K}(N q)$ and each $u_{i j} \in \mathrm{P}_{2,0}(\mathbb{Q})$. We made no attempt to optimize the computation of $\kappa$ and $\mu$, and the expense of computing the $\left(1+q+q^{2}+q^{3}\right) N \prod_{p \mid N}(1+1 / p)$ decompositions $g=\kappa u$ led us to use small values of $q$ whenever possible. On the other hand, we needed $q$ large enough to make tracing down hit the bulk of $\mathcal{S}_{2}(\mathrm{~K}(N))^{+}$. We proceeded by a mixture of experiment and feel, with the experimental attempts to span a large subspace of $\mathcal{S}_{2}(\mathrm{~K}(N))^{+}$time-consuming because tracing down ran slowly even with parallel computing. Our values of $q$ for tracing down ranged from 3 to 11 .

The trace down operator from $\mathcal{S}_{k}(\mathrm{~K}(N q))$ to $\mathcal{S}_{k}(\mathrm{~K}(N))$ is

$$
f \longmapsto \sum_{i, j} f\left[u_{i j}\right]_{k} \text {. }
$$

Fix $i$ and $j$, and let $u_{i j}=u=\left[\begin{array}{cc}d^{*} & b \\ 0 & d\end{array}\right]$. By a familiar computation, $f[u]_{k}$ has Fourier coefficients $a\left(t ; f[u]_{k}\right)=(\operatorname{det} d)^{-k} \mathrm{e}\left(\operatorname{tr}\left(t d^{\prime} b\right)\right) a\left(t\left[d^{\prime}\right] ; f\right)$, and so the Fourier coefficients of the trace down image are

$$
a(t ; \operatorname{TrDn} f)=\sum_{i, j}\left(\operatorname{det} d_{i j}\right)^{-k} \mathrm{e}\left(\operatorname{tr}\left(t d_{i j}^{\prime} b_{i j}\right)\right) a\left(t\left[d_{i j}^{\prime}\right] ; f\right) .
$$

\section{Hecke Spreading}

We used Hecke operators mainly to create elements of the Fricke minus space $\mathcal{S}_{4}(\mathrm{~K}(N))^{-}$from elements of the plus space. For this section, let $G$ denote the subspace $\operatorname{Grit}\left(\mathrm{J}_{2, N}^{\text {cusp }}\right)$ of $\mathcal{S}_{2}(\mathrm{~K}(N))^{+}$. A Hecke operator $T$ of $\mathcal{S}_{4}(\mathrm{~K}(N))$ need not respect ring structure, and so even though $T(G) \subset G$ and $G \cdot G \subset \mathcal{S}_{4}(\mathrm{~K}(N))^{+}$, the space $T(G \cdot G)$ need not lie in $\mathcal{S}_{4}(\mathrm{~K}(N))^{+}$. Indeed, for prime divisors $p$ of $N$, the operator $T\left(p^{2}\right)$ can take elements of $G \cdot G$ into $\mathcal{S}_{4}(\mathrm{~K}(N))^{-}$. Here the operators $T(n)$ for $n$ coprime to $N$ are standard ([PY15]), and we let $T(p)$ and $T\left(p^{2}\right)$ for $p \mid N$ denote the operators $T_{0,1}$ and $T_{1,0}$ of [RS07]; explicit single coset decomposition formulas for these operators appear in [PSY16]. We increased our span of $\mathcal{S}_{4}(\mathrm{~K}(N))$ with $T(G \cdot G)$ for various $T$.

Our computations represented elements of $\mathcal{S}_{4}(\mathrm{~K}(N))^{ \pm}$as vectors of Fourier coefficients indexed by $\Gamma_{ \pm}^{0}(N)$-equivalence classes in $\mathcal{X}_{2}(N)$, with a cap on the determinants of the class representatives $\left[\begin{array}{cc}n & r / 2 \\ r / 2 & m N\end{array}\right]$, i.e., $4 n m N-r^{2} \leq d$. The cap was determined from the results of Jacobi restriction on $\mathcal{S}_{4}(\mathrm{~K}(N))^{+}$, the larger of the two Fricke eigenspaces for our range of $N$-values. However, for a Hecke operator $T(n)$ to return a Fourier coefficient vector indexed by such a determinant-shell of class representatives, it needs for its input a Fourier coefficient vector indexed 
by class representatives out to determinant $n^{2} d$. Depending on various parameters, this can raise the vector length from hundreds to hundreds of thousands, or even well over a million. Computing a basis of $G \cdot G$ to so many terms was a significant computational expense, generally limiting our Hecke spreading to $T(n)$ for $n=4,8,9,12$. For $N$ coprime to 6 , Hecke spreading was not available to us as a method to span any of $\mathcal{S}_{4}(\mathrm{~K}(N))^{-}$. Also, Hecke spreading had little to start with for levels $N$ at which $G$ is small, and indeed it had nothing to start with when $G=0$. Furthermore, Hecke spreading into $\mathcal{S}_{4}(\mathrm{~K}(N))^{+}$can produce only forms having Atkin-Lehner signatures already in $G \cdot G$, and Hecke spreading seems unable to reach old forms in either Fricke eigenspace when they come from minus forms. When tracing down and Hecke spreading didn't give us enough dimensions, we searched for Borcherds products in the space, usually in the Fricke minus space but sometimes in the Fricke plus space as well. We next proceed to a discussion of these matters.

\section{Borcherds Products}

The following theorem gives sufficient conditions for a Borcherds product to be a Siegel paramodular Fricke eigenform; it is a special case of Theorem 3.3 of GPY15, which in turn is quoted from GN97, GN98, Gri12 and relies on the work of R. Borcherds. The corollary to follow will give sufficient conditions for such a Borcherds product furthermore to be a cusp form when its level is squarefree.

Theorem 7.1. Let $N$ be a positive integer. Let $\psi \in \mathrm{J}_{0, N}^{\mathrm{w} . \mathrm{h}}$. be a weakly holomorphic weight 0 , degree $N$ Jacobi form, having Fourier expansion

$$
\psi(\tau, z)=\sum_{\substack{n, r \in \mathbb{Z} \\ n \gg-\infty}} c(n, r) q^{n} \zeta^{r} \quad \text { where } q=\mathrm{e}(\tau), \zeta=\mathrm{e}(z)
$$

Define

$$
\begin{aligned}
A & =\frac{1}{24} c(0, r)+\frac{1}{12} \sum_{r \in \mathbb{Z}_{\geq 1}} c(0, r), & B & =\frac{1}{2} \sum_{r \in \mathbb{Z}_{\geq 1}} r c(0, r) \\
C & =\frac{1}{2} \sum_{r \in \mathbb{Z}_{\geq 1}} r^{2} c(0, r), & D_{0} & =\sum_{n \in \mathbb{Z}_{\leq-1}} \sigma_{0}(|n|) c(n, 0) .
\end{aligned}
$$

Suppose that the following conditions hold:

(1) $c(n, r) \in \mathbb{Z}$ for all integer pairs $(n, r)$ such that $4 n N-r^{2} \leq 0$,

(2) $A \in \mathbb{Z}$,

(3) $\sum_{j \in \mathbb{Z}_{\geq 1}} c\left(j^{2} n m, j r\right) \geq 0$ for all primitive integer triples $(n, m, r)$ such that $4 n m N-r^{2}<0$ and $m \geq 0$.

Then for weight $k=\frac{1}{2} c(0,0)$ and Fricke eigenvalue $\epsilon=(-1)^{k+D_{0}}$, the Borcherds product $\operatorname{Borch}(\psi)$ lies in $\mathcal{M}_{k}(\mathrm{~K}(N))^{\epsilon}$. For sufficiently large $\lambda$, for $\Omega=\left[\begin{array}{cc}\tau & z \\ z & \omega\end{array}\right] \in \mathcal{H}_{2}$ and $\xi=\mathrm{e}(\omega)$, the Borcherds product has the following convergent product expression on the subset $\left\{\operatorname{Im}(\Omega)>\lambda I_{2}\right\}$ of $\mathcal{H}_{2}$ :

$$
\operatorname{Borch}(\psi)(\Omega)=q^{A} \zeta^{B} \xi^{C} \prod_{\begin{array}{c}
n, m, r \in \mathbb{Z}, m \geq 0 \\
\text { if } m=0 \text { then } n \geq 0 \\
\text { if } m=n=0 \text { then } r<0
\end{array}}\left(1-q^{n} \zeta^{r} \xi^{m N}\right)^{c(n m, r)} .
$$


Also, let $\varphi(r)=c(0, r)$ for $r \in \mathbb{Z}_{\geq 0}$, and recall the corresponding theta block,

$$
\operatorname{TB}(\varphi)(\tau, z)=\eta(\tau)^{\varphi(0)} \prod_{r \in \mathbb{Z}_{\geq 1}}\left(\vartheta_{r}(\tau, z) / \eta(\tau)\right)^{\varphi(r)} \quad \text { where } \vartheta_{r}(\tau, z)=\vartheta(\tau, r z) .
$$

On $\left\{\operatorname{Im}(\Omega)>\lambda I_{2}\right\}$ the Borcherds product is a rearrangement of a convergent infinite series,

$$
\operatorname{Borch}(\psi)(\Omega)=\operatorname{TB}(\varphi)(\tau, z) \xi^{C} \exp (-\operatorname{Grit}(\psi)(\Omega)) .
$$

We make some remarks about the theorem.

- $A, B, C$, and $D_{0}$ are finite sums. Indeed, if the Fourier series expansion of $\psi$ is supported on $n \geq n_{o}$ then it is supported on $4 n N-r^{2} \geq 4 n_{o} N-N^{2}$, so especially $c(0, r)=0$ if $r^{2}>N^{2}-4 n_{0} N$. Also, conditions (1) and (3) in the theorem are finite to verify, (1) because the coefficients $c(n, r)$ where $4 n N-r^{2} \leq 0$ are determined by the coefficients such that furthermore $n \leq N / 4$ and $|r| \leq N$, and (3) because $\psi$ is supported on the pairs $(n, r)$ such that $4 n N-r^{2} \gg-\infty$.

- Condition (1) implies that $c(n, r) \in \mathbb{Z}$ for all $(n, r)$; this a general fact about weakly holomorphic Jacobi forms of weight 0 . Because the coefficients are integral but possibly negative, the theta block in the theorem could be a theta block with denominator.

- The quantity $A$ is often written as $(1 / 24) \sum_{r \in \mathbb{Z}} c(0, r)$ and the condition $A \in \mathbb{Z}$ is often phrased that $\sum_{r \in \mathbb{Z}} c(0, r)$ is an integer multiple of 24 . We phrased the theorem to make clear that the $q^{A}$ in the product expression of $\operatorname{Borch}(\psi)$ can be read off from the theta block in the series representation, as can the $\zeta^{B}$. The integrality of the coefficients and the condition $A \in \mathbb{Z}$ make $k$ integral because $c(0,0)+2 \sum_{r \in \mathbb{Z}_{>1}} c(0, r)$ is a multiple of 24 , and so $c(0,0)$ is even.

- The divisor of $\operatorname{Borch}(\psi)$ is a sum of Humbert surfaces with multiplicities, the multiplicities necessarily nonnegative for holomorphy. Let $\mathrm{K}(N)^{+}$denote the supergroup of $\mathrm{K}(N)$ obtained by adjoining the paramodular Fricke involution. The sum in item (3) is the multiplicity of the following Humbert surface in the divisor,

$$
\operatorname{Hum}\left(r^{2}-4 n m, r\right)=\mathrm{K}(N)^{+}\left\{\Omega \in \mathcal{H}_{2}:\left\langle\Omega,\left[\begin{array}{cc}
n & r / 2 \\
r / 2 & m N
\end{array}\right]\right\rangle=0\right\} .
$$

This surface lies in $\mathrm{K}(N)^{+} \backslash \mathcal{H}_{2}$. As the notation in the display suggests, this surface depends only on the discriminant $d=r^{2}-4 n m$ and on $r$, and furthermore it depends only on the residue class of $r$ modulo $2 N$; this result is due to Gritsenko and Hulek GH98. We use it to parametrize Humbert surfaces as $\operatorname{Hum}(d, r)$, taking for each such surface a suitable $\left[\begin{array}{cc}n & \tilde{r} / 2 \\ \tilde{r} / 2 & m N\end{array}\right]$ with $\operatorname{gcd}(n, m, \tilde{r})=1$ and $m \geq 0$ and $\tilde{r}^{2}-4 n m N=d$ and $\tilde{r}=r \bmod 2 N$.

- The series representation of $\operatorname{Borch}(\psi)$ in the theorem gives an experimental algorithm for the construction of holomorphic Borcherds products, based on using the series to calculate an initial portion of the Fourier-Jacobi expansion of Borch $(\psi)$. The algorithm and computer program for searching for suitable $\psi$ will be covered in an article being prepared [PYb; we used a comparatively simple part of the algorithm and program for the computation being reported in this article. Our website for this article certifies the Borcherds products that we constructed, as will be discussed below. 
- The condition $\epsilon=(-1)^{k+D_{0}}$ where $k$ is the weight says in particular that for even $k$ the Fricke eigenvalue of $\operatorname{Borch}(\psi)$ is 1 if $\psi$ has principal part 0 and is -1 if $\psi$ has principal part $1 / q$. In our computation we used such $\psi$ to create Fricke plus and minus eigenforms in spanning the spaces $\mathcal{S}_{4}(\mathrm{~K}(N))$.

Theorem 7.1 and Proposition 3.1 let us determine when a given weakly holomorphic Jacobi form of weight 0 and squarefree index $N$ gives rise through its Borcherds product to a cusp form.

Corollary 7.2. Let $N$ be a squarefree positive integer, let $k$ be an odd positive integer or let $k$ be one of $\{2,4,6,8,10,14\}$, and let $\epsilon= \pm 1$. Let $\psi, A, B, C, D_{0}$ be as in Theorem 7.1. with $k=\frac{1}{2} c(0,0)$ and $\epsilon=(-1)^{\frac{1}{2} c(0,0)+D_{0}}$. Suppose that the three conditions of the theorem hold,

(1) $c(n, r) \in \mathbb{Z}$ for all integer pairs $(n, r)$ such that $4 n N-r^{2} \leq 0$,

(2) $A \in \mathbb{Z}$

(3) $\sum_{j \in \mathbb{Z}} c\left(j^{2} n_{o} m_{o}, j r_{o}\right) \geq 0$ for all primitive integer triples $\left(n_{o}, m_{o}, r_{o}\right)$ such that $4 n_{o} m_{o} N-r_{o}^{2}<0$ and $m_{o} \geq 0$.

If $k$ is odd or $k=2$, or if $k \in\{4,6,8,10,14\}$ and $C>0$, then the Borcherds product $\operatorname{Borch}(\psi)$ of Theorem 7.1 lies in the cusp form space $\mathcal{S}_{k}(\mathrm{~K}(N))^{\epsilon}$ and its first nonzero Fourier-Jacobi coefficient has index $C>0$.

Proof. The Borcherds product $\operatorname{Borch}(\psi)$ lies in $\mathcal{M}_{k}(\mathrm{~K}(N))^{\epsilon}$. The product expression and the series representation of $\operatorname{Borch}(\psi)$ both show that its first nonzero Fourier-Jacobi coefficient has index $C$. If $k$ is odd or $k=2$ then Proposition 3.1 says that $\operatorname{Borch}(\psi)$ is a cusp form. If $k \in\{4,6,8,10,14\}$ and $C>0$ then because the Borcherds product is a multiple of $q^{A} \zeta^{B} \xi^{C}$, the constant term $a(0 ; \operatorname{Borch}(\psi))$ is 0 , and again Proposition 3.1 says that $\operatorname{Borch}(\psi)$ is a cusp form. If $\operatorname{Borch}(\psi)$ is a cusp form then the index matrix $\left[\begin{array}{cc}A & B / 2 \\ B / 2 & C\end{array}\right]$ of its term $q^{A} \zeta^{B} \xi^{C}$ must be positive, requiring $C>0$.

To create weight 4 Borcherds products for the computation being reported in this article, we used two constructions of Jacobi forms $\psi \in J_{0, N}^{\text {w.h. that }}$ the amenable to Corollary 7.2. For $\mathcal{S}_{4}(\mathrm{~K}(N))^{+}$Borcherds products, we used quotients $\psi=\psi_{12} / \Delta_{12}$ where $\psi_{12} \in \mathrm{J}_{12, N}^{\text {cusp }}$ and $\Delta_{12} \in \mathrm{J}_{12,0}^{\text {cusp }}$ is the classical discriminant function. For each level $N$ at which we used such Borcherds products $\operatorname{Borch}(\psi)$, a "BP+" file at our website Yue16] gives the singular part of each $\psi$ that we used, so that the conditions of Corollary 7.2 can be verified for it; also the file gives the leading Fourier-Jacobi coefficient $\phi$ of each Borcherds lift, and the file describes each $\psi$ as a linear combination of a basis of $\mathrm{J}_{12, N}^{\text {cusp }}$, giving the linear combination vector and then giving the basis. For $\mathcal{S}_{4}(\mathrm{~K}(N))^{-}$Borcherds products Borch $(\psi)$, a construction $\phi \mapsto \phi \mid V_{2} / \phi$ for $\phi \in \mathrm{J}_{4, N}^{\text {cusp }}$, where $V_{2}$ is an index-raising Hecke operator [EZ85], also contributes to $\psi$; this construction is central in GPY15, and it gives a weakly holomorphic Jacobi form with integral Fourier coefficients when $\phi$ is a theta block without denominator. For each level $N$ at which we used such Borcherds products, a "BP-" file at our website again gives the singular part of each $\psi$, then $\phi$, which is again the leading Fourier-Jacobi coefficient of the Borcherds product, then the linear combination vector, and then the basis. 


\section{Construction of the nOnlift newForms}

At level $N=249$ and at level $N=295$, Jacobi restriction gives a truncated Fourier-Jacobi expansion of a putative weight 2 Fricke plus space nonlift $b_{N}$ with first Fourier-Jacobi coefficient 0, i.e., $b_{N}(\Omega)=\phi_{2}\left(b_{N}\right)(\tau, z) \xi^{2 N}+\phi_{3}(\tau, z) \xi^{3 N}+\cdots$. Because the first Fourier-Jacobi coefficient is 0 , this $b_{N}$ is a nonlift in $\mathcal{S}_{2}(\mathrm{~K}(N))^{+}$ if it exists in $\mathcal{S}_{2}(\mathrm{~K}(N))^{+}$at all. We hope to show that $b_{N}$ exists in $\mathcal{S}_{2}(\mathrm{~K}(N))^{+}$by showing that it takes the form $b_{N}=\operatorname{Borch}\left(\psi_{N}\right)$ for some $\psi_{N} \in \mathrm{J}_{0, N}^{\mathrm{w} . \mathrm{h}}$, though there is no guarantee that this happens even if $b_{N}$ exists in $\mathcal{S}_{2}(\mathrm{~K}(N))^{+}$. The infinite series form of $\operatorname{Borch}\left(\psi_{N}\right)$ in Theorem 7.1 shows that necessarily $\psi_{N}=-\phi_{3} / \phi_{2}$. Thus we make an educated guess at an element $\psi_{N}$ of $\mathrm{J}_{0, N}^{\mathrm{w} . \mathrm{h}}$. that has the same initial Fourier expansion as $-\phi_{3} / \phi_{2}$ would if $\phi_{3}$ were divisible by $\phi_{2}$. If $b_{N}$ indeed exists as a Borcherds product $\operatorname{Borch}\left(\psi_{N}\right)$ and we guess $\psi_{N}$ well then Corollary 7.2 confirms that $b_{N}$ exists in $\mathcal{S}_{2}(\mathrm{~K}(N))^{+}$, and Jacobi restriction has helped us find our desired nonlift. An article being prepared on general methods for constructing nonlifts PYa will give details on how to identify a candidate weakly holomorphic weight 0 Jacobi form as arising from a quotient of dilated theta functions that demonstrably lies in $\mathrm{J}_{0, N}^{\mathrm{w} . \mathrm{h}}$. In this article we simply present $\psi_{N}$ as a product of quotients of dilated theta functions for $N=249,295$.

Consider the following product of quotients of dilated theta functions:

$$
\psi_{249}=\frac{\vartheta_{8}}{\vartheta_{1}} \frac{\vartheta_{18}}{\vartheta_{6}} \frac{\vartheta_{14}}{\vartheta_{7}} .
$$

Each quotient in the previous display takes the form $\vartheta_{d} / \vartheta_{e}$ with $e \mid d$; this divisibility makes the quotient a weakly holomorphic Jacobi form of weight 0 and index $\left(d^{2}-e^{2}\right) / 2$, and thus altogether $\psi_{249} \in \mathrm{J}_{0,249}^{\mathrm{w} . \mathrm{h}}$. The singular part of $\psi_{249}$ up to $q^{\lfloor 249 / 4\rfloor}$, which determines all of the singular part, is

$$
\begin{aligned}
4 & +\zeta^{-13}+\zeta^{-12}+\zeta^{-11}+\zeta^{-10}+\zeta^{-9}+\zeta^{-8}+2 \zeta^{-7}+3 \zeta^{-6}+2 \zeta^{-5}+2 \zeta^{-4} \\
& +2 \zeta^{-3}+2 \zeta^{-2}+3 \zeta^{-1}+3 \zeta+2 \zeta^{2}+2 \zeta^{3}+2 \zeta^{4}+2 \zeta^{5}+3 \zeta^{6}+2 \zeta^{7}+\zeta^{8} \\
& +\zeta^{9}+\zeta^{10}+\zeta^{11}+\zeta^{12}+\zeta^{13}+q^{2}\left(\zeta^{45}+\zeta^{-45}\right)+q^{5}\left(\zeta^{71}+\zeta^{-71}\right) \\
& +q^{6}\left(\zeta^{78}+\zeta^{-78}\right)+q^{7}\left(\zeta^{84}+\zeta^{-84}\right)+q^{8}\left(\zeta^{90}+\zeta^{-90}\right) \\
& +q^{24}\left(\zeta^{155}+\zeta^{-155}\right)+q^{26}\left(\zeta^{161}+\zeta^{-161}\right)+q^{28}\left(\zeta^{167}+\zeta^{-167}\right) \\
& -q^{43}\left(\zeta^{207}+\zeta^{-207}\right)+q^{54}\left(\zeta^{232}+\zeta^{-232}\right) .
\end{aligned}
$$

This $\psi_{249}$ satisfies the three conditions of Corollary 7.2 the Fourier coefficients of the singular part are integers; $A=2$; and Table 1 shows that the Humbert surface multiplicity $m_{d, r}$ is nonnegative for all possible $(d, r)$ such that any term in the $(d, r)$-multiplicity formula could be nonzero. Also we have $B=63$ and $C=498$ and $D_{0}=0$. Because $c(0,0)=4$, the corollary says that $\operatorname{Borch}\left(\psi_{249}\right)$ lies in $\mathcal{S}_{2}(\mathrm{~K}(249))^{+}$, and its first nonzero Fourier-Jacobi coefficient has index $C=2 \cdot 249$.

With the knowledge that running Jacobi restriction to five terms gives us a basis of $\mathcal{S}_{2}(\mathrm{~K}(249)$ ), we can use the expansions of the basis to compute the action of the Hecke operator $T(2)$. This operator separates the space into one-dimensional eigenspaces, with the space spanned by the nonlift eigenform $f_{249}$ readily identifiable because it doesn't lie in $\operatorname{Grit}\left(\mathrm{J}_{2,249}^{\text {cusp }}\right)$. We have the first two Jacobi coefficients of $\operatorname{Borch}\left(\psi_{249}\right)$, and this is sufficient to express $f_{249}$ as a linear combination of 
TABLE 1. Humbert surface multiplicities of $\operatorname{Borch}\left(\psi_{249}\right)$

\begin{tabular}{|c|c|}
\hline$(d, r)$ & $m_{d, r}$ \\
\hline \hline$(1,1)$ & 22 \\
\hline$(1,167)$ & 10 \\
\hline$(3,2)$ & 10 \\
\hline$(4,164)$ & 4 \\
\hline$(9,3)$ & 7 \\
\hline$(12,192)$ & 1 \\
\hline$(16,4)$ & 4 \\
\hline$(16,170)$ & 1 \\
\hline$(21,207)$ & 0 \\
\hline
\end{tabular}

\begin{tabular}{|c|c|}
\hline$(d, r)$ & $m_{d, r}$ \\
\hline \hline$(25,5)$ & 3 \\
\hline$(25,161)$ & 1 \\
\hline$(33,45)$ & 2 \\
\hline$(36,6)$ & 4 \\
\hline$(40,232)$ & 1 \\
\hline$(49,7)$ & 2 \\
\hline$(61,71)$ & 1 \\
\hline$(64,8)$ & 1 \\
\hline$(81,9)$ & 1 \\
\hline
\end{tabular}

\begin{tabular}{|c|c|}
\hline$(d, r)$ & $m_{d, r}$ \\
\hline \hline$(84,84)$ & 1 \\
\hline$(100,10)$ & 1 \\
\hline$(108,78)$ & 1 \\
\hline$(121,11)$ & 1 \\
\hline$(121,155)$ & 1 \\
\hline$(132,90)$ & 1 \\
\hline$(144,12)$ & 1 \\
\hline$(169,13)$ & 1 \\
\hline & \\
\hline
\end{tabular}

$\operatorname{Borch}\left(\psi_{249}\right)$ and a Gritsenko lift,

$$
\begin{aligned}
f_{249}= & 14 \operatorname{Borch}\left(\psi_{249}\right) \\
& -6 \operatorname{Grit}(\operatorname{TB}(2 ; 2,3,3,4,5,6,7,9,10,13)) \\
& -3 \operatorname{Grit}(\operatorname{TB}(2 ; 2,2,3,5,5,6,7,9,11,12)) \\
& +3 \operatorname{Grit}(\operatorname{TB}(2 ; 1,3,3,5,6,6,6,9,11,12)) \\
& +2 \operatorname{Grit}(\operatorname{TB}(2 ; 1,1,2,3,4,5,6,9,10,15)) \\
& +7 \operatorname{Grit}(\operatorname{TB}(2 ; 1,2,3,3,4,5,6,9,11,14)) .
\end{aligned}
$$

Here $\operatorname{TB}(2 ; 2,3,3,4,5,6,7,9,10,13)$ is the weight 2 theta block $\operatorname{TB}(\varphi)$ where $\varphi(r)=$ 1 for $r=2,4,5,6,7,9,10,13$ and $\varphi(3)=2$ and $\varphi(r)=0$ for all other $r \in \mathbb{Z}_{\geq 1}$, and similarly for the other theta blocks. Many Fourier coefficients of $f_{249}$ are given at our website Yue16. Thus $f_{249}$ is congruent to a lift modulo 14, and as noted earlier in this article, the two isogenous Jacobians of genus 2 curves of conductor 249 defined over $\mathbb{Q}$ have torsion groups $\mathbb{Z} / 14 \mathbb{Z}$ and $\mathbb{Z} / 28 \mathbb{Z}$.

Because $\mathcal{S}_{2}(\mathrm{~K}(3))=0$, and because $\mathcal{S}_{2}(\mathrm{~K}(83))$ is spanned by Gritsenko lifts and level-raising operators take lifts to lifts, the nonlift eigenform $f_{249}$ is in fact a newform.

To compute the $T(n)$-action on $f_{249}$ for $n \leq 25$ coprime to 249 , we expanded $f_{249}$ to 25 Fourier-Jacobi coefficients. The relevant eigenvalues are $\lambda_{2}=-2$ and $\lambda_{4}=0$, $\lambda_{5}=0$ and $\lambda_{25}=-3, \lambda_{7}=-1, \lambda_{11}=1, \lambda_{13}=0, \lambda_{17}=-1, \lambda_{19}=-6, \lambda_{23}=0$. These eigenvalues determine the spin $p$-Euler factor for $p=2,5$, because in general for $p \nmid N$ the spin $p$-Euler factor for weight 2 is (see section 5.3 of [And09, for example)

$$
1-\lambda_{p} T+\left(\lambda_{p}^{2}-\lambda_{p^{2}}-1\right) T^{2}-\lambda_{p} p T^{3}+p^{2} T^{4} .
$$

The spin 2-Euler factor and the spin 5-Euler factor of $f_{249}$ are

$$
1+2 T+3 T^{2}+4 T^{3}+4 T^{4}, \quad 1+2 T^{2}+25 T^{4},
$$

and the reader can check at the online database lmfdb.org that these Euler factors match those of the genus 2 curve class 249.a. The Jacobian of this curve is an abelian surface of conductor 249 defined over $\mathbb{Q}$, and it has the same Euler factors. The spin $p$-Euler factors of $f_{249}$ for $p=7,11,13,17,19,23$ are also compatible with the 249.a $L$-factors at $1 \mathrm{mfdb}$.org, with a match depending on the uncomputed $\lambda_{p^{2}}$ in each case. 
TABLE 2. Humbert surface multiplicities of $\operatorname{Borch}\left(\psi_{295}\right)$

\begin{tabular}{|c|c|}
\hline$(d, r)$ & $m_{d, r}$ \\
\hline \hline$(1,1)$ & 22 \\
\hline$(1,119)$ & 10 \\
\hline$(4,2)$ & 10 \\
\hline$(4,238)$ & 4 \\
\hline$(5,185)$ & 3 \\
\hline$(9,3)$ & 6 \\
\hline$(9,233)$ & 2 \\
\hline$(16,4)$ & 4 \\
\hline$(16,114)$ & 2 \\
\hline$(20,220)$ & 1 \\
\hline
\end{tabular}

\begin{tabular}{|c|c|}
\hline$(d, r)$ & $m_{d, r}$ \\
\hline \hline$(21,91)$ & 0 \\
\hline$(21,209)$ & 0 \\
\hline$(25,5)$ & 4 \\
\hline$(36,6)$ & 2 \\
\hline$(41,69)$ & 1 \\
\hline$(49,7)$ & 2 \\
\hline$(64,8)$ & 2 \\
\hline$(64,228)$ & 1 \\
\hline$(76,264)$ & 1 \\
\hline$(81,9)$ & 1 \\
\hline
\end{tabular}

\begin{tabular}{|c|c|}
\hline$(d, r)$ & $m_{d, r}$ \\
\hline \hline$(81,109)$ & 1 \\
\hline$(84,172)$ & 1 \\
\hline$(84,182)$ & 1 \\
\hline$(100,10)$ & 2 \\
\hline$(121,11)$ & 1 \\
\hline$(169,13)$ & 1 \\
\hline$(169,223)$ & 1 \\
\hline$(181,179)$ & 1 \\
\hline$(256,16)$ & 1 \\
\hline & \\
\hline
\end{tabular}

Similarly, consider

$$
\psi_{295}=\frac{\vartheta_{12}}{\vartheta_{3}} \frac{\vartheta_{15}}{\vartheta_{5}} \frac{\vartheta_{12}}{\vartheta_{6}} \frac{\vartheta_{14}}{\vartheta_{7}} .
$$

Here $\psi_{295}$ lies in $\mathrm{J}_{0,295}^{\mathrm{w} . h}$, and its singular part up to $q^{\lfloor 295 / 4\rfloor}$ is

$$
\begin{aligned}
4 & +\zeta^{-16}+\zeta^{-13}+\zeta^{-11}+2 \zeta^{-10}+\zeta^{-9}+\zeta^{-8}+2 \zeta^{-7}+2 \zeta^{-6}+2 \zeta^{-5}+2 \zeta^{-4} \\
& +3 \zeta^{-3}+2 \zeta^{-2}+2 \zeta^{-1}+2 \zeta+2 \zeta^{2}+3 \zeta^{3}+2 \zeta^{4}+2 \zeta^{5}+2 \zeta^{6}+2 \zeta^{7}+\zeta^{8} \\
& +\zeta^{9}+2 \zeta^{10}+\zeta^{11}+\zeta^{13}+\zeta^{16}+q^{4}\left(\zeta^{69}+\zeta^{-69}\right)-q^{7}\left(\zeta^{91}+\zeta^{-91}\right) \\
& +q^{10}\left(\zeta^{109}+\zeta^{-109}\right)+q^{11}\left(\zeta^{114}+\zeta^{-114}\right)+q^{12}\left(\zeta^{119}+\zeta^{-119}\right) \\
& +q^{25}\left(\zeta^{172}+\zeta^{-172}\right)+q^{27}\left(\zeta^{179}+\zeta^{-179}\right)+q^{28}\left(\zeta^{182}+\zeta^{-182}\right) \\
& +2 q^{29}\left(\zeta^{185}+\zeta^{-185}\right)-q^{37}\left(\zeta^{209}+\zeta^{-209}\right)+q^{41}\left(\zeta^{220}+\zeta^{-220}\right) \\
& +q^{42}\left(\zeta^{223}+\zeta^{-223}\right)+q^{44}\left(\zeta^{228}+\zeta^{-228}\right)+q^{46}\left(\zeta^{233}+\zeta^{-233}\right) \\
& +q^{59}\left(\zeta^{264}+\zeta^{-264}\right) .
\end{aligned}
$$

This $\psi_{295}$ satisfies the three conditions of Corollary 7.2 . similarly to $\psi_{249}$; here the Humbert surface multiplicities are given in Table 2, In this case, $A=2$ and $B=68$ and $C=590$ and $D_{0}=0$. Again $c(0,0)=4$, so $\operatorname{Borch}\left(\psi_{295}\right)$ lies in $\mathcal{S}_{2}(\mathrm{~K}(295))^{+}$ and its first nonzero Fourier-Jacobi coefficient has index $C=2 \cdot 295$.

The Hecke operator $T(2)$ separates $\mathcal{S}_{2}(\mathrm{~K}(295))$ into one-dimensional eigenspaces, and we can identify the nonlift eigenform $f_{295}$ and then express it as a linear combination of $\operatorname{Borch}\left(\psi_{295}\right)$ and a Gritsenko lift,

$$
\begin{aligned}
f_{295}= & 7 \operatorname{Borch}\left(\psi_{295}\right) \\
& +4 \operatorname{Grit}(\operatorname{TB}(2 ; 1,1,2,3,3,4,5,10,13,16)) \\
& +\operatorname{Grit}(\operatorname{TB}(2 ; 1,1,2,3,4,7,8,10,11,15)) \\
& -2 \operatorname{Grit}(\operatorname{TB}(2 ; 2,3,4,4,6,7,8,10,10,14)) \\
& -\operatorname{Grit}(\operatorname{TB}(2 ; 1,1,5,6,7,8,8,9,10,13)) \\
& -3 \operatorname{Grit}(\operatorname{TB}(2 ; 1,2,3,4,5,5,8,9,13,14)),
\end{aligned}
$$

and many Fourier coefficients of $f_{295}$ are at our website. Here the nonlift eigenform is congruent to a lift modulo 7 , and one of the two isogenous Jacobians of genus 2 curves of conductor 295 defined over $\mathbb{Q}$ has torsion group $\mathbb{Z} / 7 \mathbb{Z}$, as 
shown at $1 \mathrm{mfdb}$.org. The nonlift eigenform $f_{295}$ is a newform because $\mathcal{S}_{2}(\mathrm{~K}(5))=$ $\mathcal{S}_{2}(\mathrm{~K}(59))=0$. This time, after expanding $f_{295}$ to sixteen Fourier-Jacobi coefficients, our computed Hecke eigenvalues of $f_{295}$ are $\lambda_{2}=-2$ and $\lambda_{4}=0, \lambda_{3}=-1$ and $\lambda_{9}=0, \lambda_{7}=1, \lambda_{11}=2, \lambda_{13}=-2$, excluding $\lambda_{5}$ because 5 divides 295. The spin 2-Euler factor and the spin 3-Euler factor of $f_{295}$ are

$$
1+2 T+3 T^{2}+4 T^{3}+4 T^{4}, \quad 1+T+3 T^{3}+9 T^{4},
$$

and these match the corresponding Euler factors of the genus 2 curve isogeny class 295.a at Imfdb.org. The Jacobian of this curve is an abelian surface of conductor 295, having the same Euler factors. The spin $p$-Euler factors of $f_{295}$ for $p=7,11,13$ are also compatible with the 295.a $L$-factors at $1 \mathrm{mfdb}$.org, with a match depending on the uncomputed $\lambda_{p^{2}}$ in each case.

\section{REFERENCES}

[And09] Anatoli Andrianov. Introduction to Siegel modular forms and Dirichlet series. Universitext. Springer, New York, 2009.

[BK14] Armand Brumer and Kenneth Kramer. Paramodular abelian varieties of odd conductor. Trans. Amer. Math. Soc., 366(5):2463-2516, 2014.

[BPY16] Jeffery Breeding, II, Cris Poor, and David S. Yuen. Computations of spaces of paramodular forms of general level. J. Korean Math. Soc., 53(3):645-689, 2016.

[EZ85] Martin Eichler and Don Zagier. The theory of Jacobi forms, volume 55 of Progress in Mathematics. Birkhäuser Boston, Inc., Boston, MA, 1985.

[GH98] Valeri A. Gritsenko and Klaus Hulek. Minimal Siegel modular threefolds. Math. Proc. Cambridge Philos. Soc., 123(3):461-485, 1998.

[GN97] Valeri A. Gritsenko and Viacheslav V. Nikulin. Siegel automorphic form corrections of some Lorentzian Kac-Moody Lie algebras. Amer. J. Math., 119(1):181-224, 1997.

[GN98] Valeri A. Gritsenko and Viacheslav V. Nikulin. Automorphic forms and Lorentzian KacMoody algebras. II. Internat. J. Math., 9(2):201-275, 1998.

[GPY15] Valeri A. Gritsenko, Cris Poor, and David S. Yuen. Borcherds products everywhere. J. Number Theory, 148:164-195, 2015.

[GPY16] Valeri A. Gritsenko, Cris Poor, and David S. Yuen. Antisymmetric paramodular forms of weights 2 and 3, 2016. https://arxiv.org/abs/1609.04146

[Gri12] Valeri A. Gritsenko. 24 faces of the Borcherds modular form $\phi_{12}$. arXiv:1203.6503, 2012.

[GSZ] Valeri A. Gritsenko, Nils-Peter Skoruppa, and Don Zagier. Theta blocks. In preparation.

[Ibu85] Tomoyoshi Ibukiyama. On relations of dimensions of automorphic forms of $\operatorname{Sp}(2, \mathbf{R})$ and its compact twist $\mathrm{Sp}(2)$. I. In Automorphic forms and number theory (Sendai, 1983), volume 7 of Adv. Stud. Pure Math., pages 7-30. North-Holland, Amsterdam, 1985.

[Ibu93] Tomoyoshi Ibukiyama. On some alternating sum of dimensions of Siegel cusp forms of general degree and cusp configurations. J. Fac. Sci. Univ. Tokyo Sect. IA Math., 40(2):245-283, 1993.

[Ibu07] Tomoyoshi Ibukiyama. Dimension formulas of Siegel modular forms of weight 3 and supersingular abelian varieties. In Siegel Modular Forms and Abelian Varieties, Proceedings of the 4 th Spring Conference on Modular Forms and Related Topics, pages 39-60, 2007.

[IK] Tomoyoshi Ibukiyama and Hidetaka Kitayama. Dimension formulas of paramodular forms of squarefree level and comparison with inner twist. To appear in Journal of the Mathematical Society of Japan.

[IPY13] Tomoyoshi Ibukiyama, Cris Poor, and David S. Yuen. Jacobi forms that characterize paramodular forms. Abh. Math. Semin. Univ. Hambg., 83(1):111-128, 2013.

[PSY16] Cris Poor, Ralf Schmidt, and David S. Yuen. Paramodular forms of level 8 and weights 10 and 12, 2016. preprint.

[PYa] Cris Poor and David S. Yuen. Constructing paramodular nonlift newforms via Borcherds products. In preparation.

[PYb] Cris Poor and David S. Yuen. Finding all Borcherds products of a given weight and level. In preparation. 
[PY13] Cris Poor and David S. Yuen. The cusp structure of the paramodular groups for degree two. J. Korean Math. Soc., 50(2):445-464, 2013.

[PY15] Cris Poor and David S. Yuen. Paramodular cusp forms. Math. Comp., 84(293):1401$1438,2015$.

[Ree73] Helmut Reefschläger. Berechnung der Anzahl der 1-Spitzen der Paramodularen Gruppen 2-ten Grades. PhD thesis, Georg-August-Universität zu Göttingen, 1973.

[RS07] Brooks Roberts and Ralf Schmidt. Local Newforms for GSp(4), volume 1918 of Lecture Notes in Mathematics. Springer, Berlin, 2007.

[SZ89] Nils-Peter Skoruppa and Don Zagier. A trace formula for Jacobi forms. J. Reine Angew. Math., 393:168-198, 1989.

[Yue15] David S. Yuen. Modularity of the abelian surface of conductor 277, 2015. https://icerm.brown.edu/video_archive/\#/play/779

[Yue16] David S. Yuen. Degree 2 Siegel paramodular forms of weight 2 and squarefree composite level, 2016. http://siegelmodularforms.org/pages/degree2/paramodular-wt2-sqfree/

Dept. of Mathematics, Fordham University, Bronx, NY 10458 USA

E-mail address: poor@fordham.edu

Reed College, Portland, OR 97202 USA

E-mail address: jerry@reed.edu

Dept. of Mathematics and Computer Science, Lake Forest College, 555 N. Sheridan RD., LAKE Forest, IL 60045 USA

E-mail address: yuen@lakeforest.edu 